\title{
Acute dietary fat intake initiates alterations in energy metabolism and insulin resistance
}

\author{
Elisa Álvarez Hernández, ${ }^{1,2}$ Sabine Kahl, ${ }^{1,2,3}$ Anett Seelig, ${ }^{4}$ Paul Begovatz, ${ }^{1,2}$ Martin Irmler, ${ }^{5}$ Yuliya Kupriyanova, ${ }^{1,2}$ \\ Bettina Nowotny, ${ }^{1,2,3}$ Peter Nowotny, ${ }^{1,2}$ Christian Herder, ${ }^{1,2}$ Cristina Barosa, ${ }^{6}$ Filipa Carvalho, Jan Rozman, ${ }^{4,5}$ Susanne Neschen, ${ }^{2,4,5}$ \\ John G. Jones, ${ }^{6,7}$ Johannes Beckers, ${ }^{2,5,8}$ Martin Hrabě de Angelis, ${ }^{2,5,8}$ and Michael Roden ${ }^{1,2,3}$ \\ ${ }^{1}$ Institute for Clinical Diabetology, German Diabetes Center, Leibniz Center for Diabetes Research at Heinrich Heine University, Düsseldorf, Cermany. ${ }^{2}$ Cerman Center for Diabetes Research, Munich-Neuherberg, \\ Germany. ${ }^{3}$ Department of Endocrinology and Diabetology, Medical Faculty, Heinrich-Heine University Düsseldorf, Düsseldorf, Germany. ${ }^{4}$ Helmholtz Zentrum Munich, German Research Center for Environmental \\ Health (GmbH), Neuherberg, Germany. ${ }^{5}$ Institute of Experimental Genetics, Helmholtz Zentrum Munich, German Research Center for Environmental Health (GmbH), Neuherberg, Germany. ${ }^{6}$ Center for \\ Neurosciences and Cell Biology, UC Biotech, Cantanhede, Portugal. ${ }^{7}$ Portuguese Diabetes Association (APDP), Lisbon, Portugal. ${ }^{8}$ Technische Universität Munich, Chair of Experimental Genetics, Freising, Cermany.
}

BACKGROUND. Dietary intake of saturated fat is a likely contributor to nonalcoholic fatty liver disease (NAFLD) and insulin resistance, but the mechanisms that initiate these abnormalities in humans remain unclear. We examined the effects of a single oral saturated fat load on insulin sensitivity, hepatic glucose metabolism, and lipid metabolism in humans. Similarly, initiating mechanisms were examined after an equivalent challenge in mice.

METHODS. Fourteen lean, healthy individuals randomly received either palm oil (PO) or vehicle (VCL). Hepatic metabolism was analyzed using in vivo ${ }^{13} \mathrm{C} /{ }^{31} \mathrm{P} /{ }^{1} \mathrm{H}$ and ex vivo ${ }^{2} \mathrm{H}$ magnetic resonance spectroscopy before and during hyperinsulinemiceuglycemic clamps with isotope dilution. Mice underwent identical clamp procedures and hepatic transcriptome analyses.

RESULTS. PO administration decreased whole-body, hepatic, and adipose tissue insulin sensitivity by $25 \%$, $15 \%$, and $34 \%$, respectively. Hepatic triglyceride and ATP content rose by $35 \%$ and $16 \%$, respectively. Hepatic gluconeogenesis increased by $70 \%$, and net glycogenolysis declined by $20 \%$. Mouse transcriptomics revealed that PO differentially regulates predicted upstream regulators and pathways, including LPS, members of the TLR and PPAR families, NF- $\mathrm{KB}$, and TNF-related weak inducer of apoptosis (TWEAK).

CONCLUSION. Saturated fat ingestion rapidly increases hepatic lipid storage, energy metabolism, and insulin resistance. This is accompanied by regulation of hepatic gene expression and signaling that may contribute to development of NAFLD.

RECISTRATION. ClinicalTrials.gov NCT01736202.

FUNDING. Germany: Ministry of Innovation, Science, and Research North Rhine-Westfalia, German Federal Ministry of Health, Federal Ministry of Education and Research, German Center for Diabetes Research, German Research Foundation, and German Diabetes Association. Portugal: Portuguese Foundation for Science and Technology, FEDER - European Regional Development Fund, Portuguese Foundation for Science and Technology, and Rede Nacional de Ressonância Magnética Nuclear.

\section{Introduction}

The pandemic of obesity, type 2 diabetes mellitus (T2DM) and nonalcoholic fatty liver disease (NAFLD) has frequently been associated with dietary intake of saturated fats (1) and specifically with dietary palm oil (PO) (2). According to current paradigms, chronic insulin resistance is the common feature of these diseases $(3,4)$ and relates to intracellular concentrations of triglycerides (TG) and lipotoxins

\section{Related Commentary: p. 454}

Authorship note: E. Álvarez Hernández, S. Kahl, A. Seelig, and P. Begovatz contributed equally to this work.

Conflict of interest: The authors have declared that no conflict of interest exists.

Submitted: July 27, 2016; Accepted: November 10, 2016.

Reference information: J Clin Invest. 2017;127(2):695-708.

https://doi.org/10.1172/JCl89444.
(5). There is evidence that a chronic high-fat diet in mice and humans leads to insulin resistance via similar mechanisms $(6,7)$. Chronic insulin resistance comprises not only impaired muscle insulin action but also increased rates of endogenous glucose production (EGP) and gluconeogenesis (GNG) in obese and T2DM patients (8-10).

Still, only a few studies have addressed the initial effects of high-fat loading using either intravenous or enteral administration of lipids. These studies mainly focused on the role of skeletal muscle by assessing intramyocellular TG content (11), substrate oxidation, glycogen synthesis (12), or glucose disposal (13-17). Studies using parenteral administration of unsaturated lipids (18) or high-calorie mixed meals yielded conflicting results with regard to hepatic energy metabolism. One mixed-meal study found greater de novo lipogenesis without affecting hepatic glycogen metabolism (12), while an intravenous lipid infusion study failed to detect any effect on hepatic insulin sensitivity (19). Another study com- 


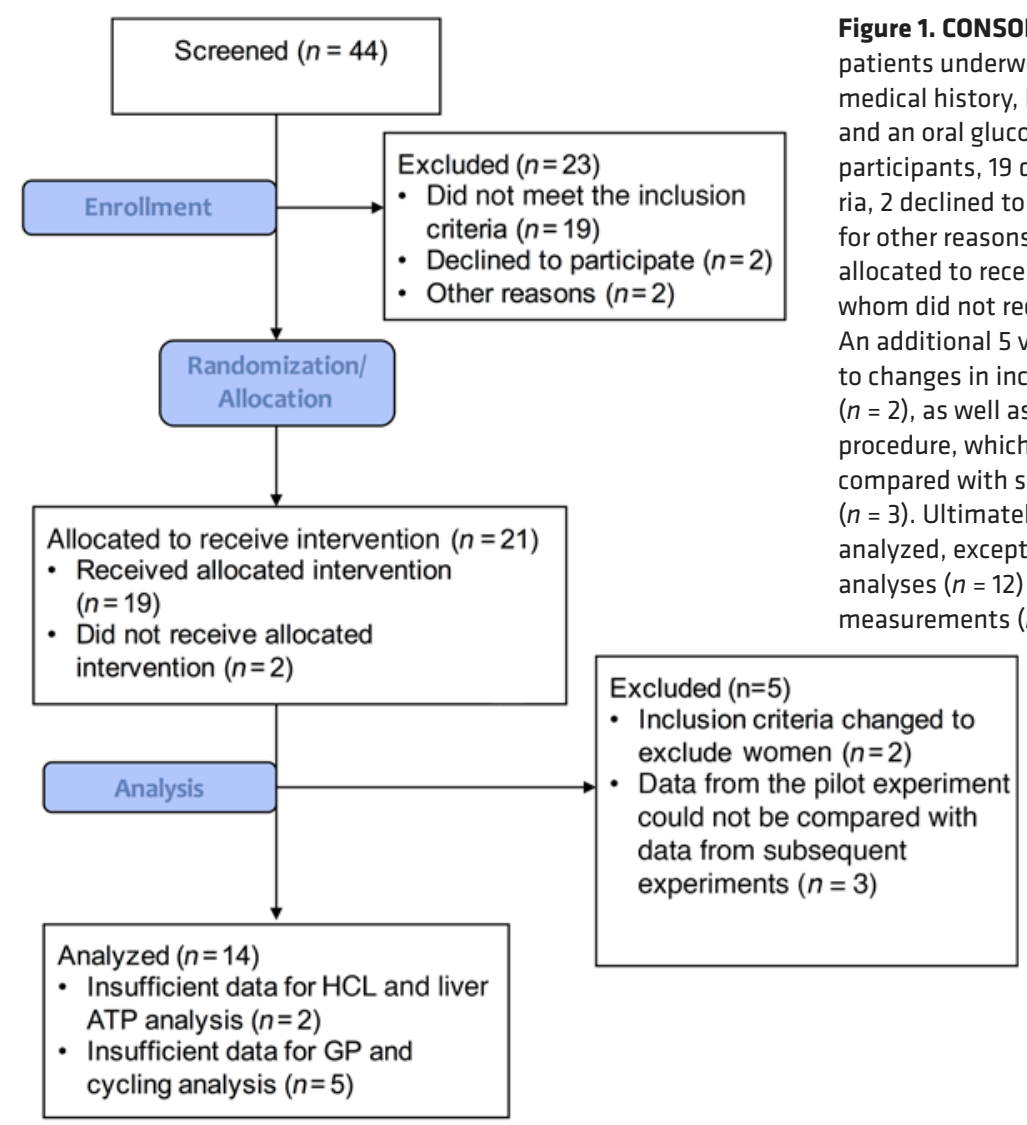

paring subacute oral ingestion of fatty acids with different compositions found an increase in the glucose infusion rate only after polyunsaturated fatty acid ingestion (20).

To overcome possible limitations of the previous studies, such as the use of nonphysiological routes of lipid administration or mixed meals, which introduce protein and carbohydrates as confounders, we designed a translational study concept comprising a clinical trial involving healthy humans and a corresponding study involving nonobese nondiabetic mice. The randomized crossover clinical trial examined the effects of a single oral challenge with PO, which is mainly composed of saturated fatty acids (2), versus vehicle (VCL) ingestion on whole-body insulin sensitivity (WBIS) and hepatic insulin sensitivity. Moreover, the contributions of hepatic glucose fluxes, i.e., GNG, net glycogenolysis (GLYnet), and the futile exchange between glycogenogenic and glycogenolytic pathways (glycogen cycling) to EGP as well as the effects of these fluxes on hepatocellular lipids (HCL) and phosphorouscontaining metabolites were analyzed using combined in vivo multinuclear ${ }^{13} \mathrm{C} /{ }^{31} \mathrm{P} /{ }^{1} \mathrm{H}$ and stable isotope tracers to assess plasma glucose appearance rates and sources of EGP. In the mouse study, we examined the effects of a similar oral saturated fat challenge on insulin sensitivity and hepatic transcriptome changes.

\section{Results}

Studies in humans. A total of 14 young, lean, insulin-sensitive male volunteers (Figure 1 and Table 1 ) received either an oral dose of PO $(\sim 1.18 \mathrm{~g} / \mathrm{kg} \mathrm{BW})$ or an identical volume of VCL on 2 occasions in random order, spaced by an 8-week interval.
$P O$ results in increased circulating TG, glucagon, and incretins. After PO administration, TG in plasma rose by $59 \%$ (area under the time curve [AUC], $P<0.001$ ) and by $156 \%$ in chylomicrons (AUC, $P=0.009$ ) (Figure 2A). The AUC for plasma free fatty acids (FFA) (Figure 2B) and insulin concentrations (Figure 2C) was unchanged, while the AUC for plasma C-peptide was $28 \%$ higher after PO ingestion versus VCL $(P<0.005$, Figure 2D). Of note, FFA were increased at 300,420 , and 480 minutes. Blood glucose levels were not different between PO- and VCL-treated groups (Figure 2E). Plasma glucagon rose by $41 \%$ (AUC, $P<0.0001$ ) only after PO ingestion (Figure $2 \mathrm{~F}$ ). Also, glucagon-like peptide 1 (GLP-1) and gastric inhibitory polypeptide (GIP) levels were markedly increased and remained elevated after PO ingestion (both $P<0.005$ ) (Supplemental Figure 2; supplemental material available online with this article; https://oi.org/10.1172/ JCI89444DS1). Circulating levels of TNF- $\alpha$, IL-6, fetuin-A, chemerin, omentin, and cortisol were not different between PO and VCL groups ( $P>0.5$ for all) (Supplemental Table 1$)$.

$P O$ induces insulin resistance at whole-body, liver, and adipose tissue levels. Insulin sensitivity was measured using hyperinsulinemic-euglycemic clamp tests in healthy humans. Steady state was reached (Supplemental Figure 1), and pertinent parameters were analyzed during this time. PO ingestion reduced WBIS by $25 \%$ compared with VCL treatment $(P=0.0005$, Figure $3 A)$. Furthermore, after PO, volunteers also showed a decrease of $22 \%$ $(P=0.002)$ in the rate of glucose disappearance $(\mathrm{Rd})$, mostly due to a $33 \%(P=0.01)$ reduction in glucose oxidation (GOX), while the rate of nonoxidative glucose disposal remained unchanged 
(Figure 3B). EGP increased by $10 \%(P=0.03)$ at 240 minutes and by $82 \%(P=0.008)$ at 480 minutes after PO compared with VCL treatment (Figure 3C). After PO, insulin-stimulated suppression of EGP was $15 \%$ lower than that detected after VCL $(P<0.01$, Figure 3D). Finally, insulin-stimulated suppression of FFA (Figure 3E) and TG (Figure $3 \mathrm{~F})$ was $30 \%(P=0.0001)$ and $80 \%(P=0.026)$ lower after $\mathrm{PO}$ than after VCL treatment.

$P O$ augments the contribution of GNG to EGP. In order to further analyze the PO-induced increase in EGP, we measured the contributions of gluconeogenic and glycogenolytic fluxes. We found that GNG increased by approximately $70 \%(P=0.01)$, while GLYnet decreased by approximately $20 \%(P<0.05)$ after PO. Glycogen phosphorylase (GP) flux tended to be lower in the PO arm $(P=0.085)$. The contribution of glycogen cycling to total GP flux was negligible in both study arms (Figure 4).

$P O$ increases lipid oxidation rates. The respiratory quotient (RQ), defined as the rate of $\mathrm{CO}_{2}$ production $/ \mathrm{O}_{2}$ consumption, was comparable in the period after PO or VCL ingestion but was reduced during the clamp only after PO (Figure 5A). Resting energy expenditure (REE) and lipid oxidation (LOX) rates increased markedly at 300 minutes after PO ingestion and remained elevated into the clamp (at $420 \mathrm{~min}$ ) after PO (Figure 5, B and C). GOX decreased during the clamp following PO ingestion compared with GOX after VCL ingestion (Figure 5D).

$P O$ raises hepatic ATP and lipid content. HCL and hepatic $\gamma$ ATP increased by $33 \%(P<0.01)$ and $16 \%(P=0.009)$ from -120 minutes to 240 minutes after PO, respectively. At 240 minutes, $\gamma$ ATP tended to be higher in the VCL arm $(P=0.06)$, while HCL levels did not differ between PO and VCL arms. Hepatic inorganic phosphate $\left(\mathrm{P}_{\mathrm{i}}\right)$ and $\gamma \mathrm{ATP} / \mathrm{P}_{\mathrm{i}}$ did not change after PO or VCL (Table 2). When comparing the mean difference between -120 and 240 minutes after PO or VCL between interventions, only the change in HCL levels tended to be increased ( $\Delta \% \mathrm{HCL}$ PO vs. VCL, $P=0.085 ; \Delta \gamma$ ATP,$\left.P=0.57 ; \Delta \mathrm{P}_{\mathrm{i}}, P=0.14 ; \Delta \gamma \mathrm{ATP} / \mathrm{P}_{\mathrm{i}}, P=0.22\right)$.

Studies in mice. Two mouse cohorts received PO or VCL via gavage. One cohort then underwent hyperinsulinemic-euglycemic clamp tests under unrestrained conditions, whereas tissue and blood sample analysis was done for the other cohort.

PO increases circulating lipid levels. Plasma FFA levels increased

Table 1. Anthropometric and blood parameters of study participants

$\begin{array}{lc}\text { Parameter } & \text { Mean } \pm \text { SEM } \\ n(\text { men/women) } & 14(14 / 0) \\ \text { Age }(\mathrm{yr}) & 25.8 \pm 1.4 \\ \text { BMI }\left(\mathrm{kg} / \mathrm{m}^{2}\right) & 22.5 \pm 0.3 \\ \text { Waist circumference }(\mathrm{cm}) & 79.5 \pm 1.4 \\ \text { Lean BW }(\mathrm{kg}) & 59.2 \pm 2.1 \\ \text { TC (mg/dl) } & 78.4 \pm 9.8 \\ \text { FFA ( } \mu \mathrm{mol} / \mathrm{l}) & 386.9 \pm 32.9 \\ \text { ALT }(\mathrm{U} / \mathrm{l}) & 24.4 \pm 3.2 \\ \text { AST }(\mathrm{U} / \mathrm{l}) & 26.0 \pm 2.5 \\ \text { Fasting blood glucose (mg/dl) } & 76.1 \pm 2.0 \\ \text { 2-hour postprandial blood glucose (mg/dl) } & 76.4 \pm 4.7 \\ \text { HCL }\left(\% \mathrm{H}_{2} \mathrm{O}\right) & 0.60 \pm 0.09 \\ \text { ALT, alanine aminotransferase; AST, aspartate. } & \end{array}$

by $45 \%$ (AUC, $P=0.004$ ), while TG levels were not significantly different (AUC, $P=0.08$ ) after PO (Figure 6, A and B). The postintervention AUC for blood glucose and insulin was comparable between groups (Figure 6, C and D).

$P O$ preferably induces hepatic insulin resistance. Insulin sensitivity was measured using hyperinsulinemic-euglycemic clamp tests in unrestrained mice. WBIS in mice trended toward a reduction $(P=0.07)$ (Figure 7A). Whole-body glucose uptake, given by Rd, was not different between interventions (Figure 7B). Residual EGP during the clamp tended to be higher $(P=0.06)$ (Figure $7 C)$, and insulin-mediated suppression of EGP was $25 \%$ lower $(P=0.04)$ after PO (Figure 7D), reflecting marked hepatic insulin resistance. PO ingestion did not affect insulin-induced FFA suppression (Figure 7E) but reduced insulin-induced TG suppression by $83 \%$ $(P=0.0039)$ (Figure 7F). Of note, glucose uptake in the gastrocnemius muscle (Supplemental Figure 3A) and white adipose tissue (Supplemental Figure 3B) as well as rates of glycolysis did not differ between the PO- and VCL-treated groups (Supplemental Figure $3 \mathrm{C}$ ). Interestingly, PO administration did not change hepatic TG content in mice (Supplemental Figure 3D).

PO causes changes in the expression of hepatic transcription factors. Livers from both mouse cohorts were used for analysis on Affymetrix Gene 2.1 ST microarrays. A total of 273 and 327 differentially regulated probe sets were obtained from each cohort. Array data were deposited in the NCBI's Gene Expression Omnibus (GEO) database (GEO GSE80259). Canonical pathways predicted to be differentially regulated by Ingenuity software after PO included TNF-like weak inducer of apoptosis (TWEAK) and aryl hydrocarbon receptor (AHR) under insulin-stimulated and noninsulin-stimulated conditions; phospholipase and D-myoinositol-5 phosphate signaling under insulin-stimulated conditions; and p38 MAPK, NF- $\mathrm{B}, \mathrm{PPAR} \alpha$, and OX40 under noninsulin-stimulated conditions (Figure 8, A and $\mathrm{B}$, all $P<0.05)$. Several upstream regulators involved in hepatic fatty acid metabolism and inflammatory processes were predicted by Ingenuity software to be regulated by PO ingestion. These upstream regulators included LPS, which was activated with the most certainty, with a $Z$ score of 1.9 and 2.3 under insulin- and noninsulin-stimulated conditions, respectively, TLR family members (TLR9 and TLR3 under insulin-stimulated conditions as well as TLR4 under noninsulin-stimulated conditions), PPAR $\alpha$, and FOXO1 (all $P<0.01$ ) (Figure 8, C and D).

Table 2. Hepatocellular lipids and hepatic phosphoruscontaining metabolites

\begin{tabular}{|c|c|c|c|c|}
\hline Time (min) & \multicolumn{2}{|c|}{-120} & \multicolumn{2}{|c|}{240} \\
\hline Group & VCL & PO & VCL & PO \\
\hline $\mathrm{HCL}\left(\% \mathrm{H}_{2} \mathrm{O}\right)$ & $0.97 \pm 0.20$ & $0.93 \pm 0.23$ & $1.04 \pm 0.21$ & $1.26 \pm 0.32^{A, D}$ \\
\hline$\gamma \operatorname{ATP}(\mathrm{mmol} / \mathrm{l})$ & $2.86 \pm 0.17$ & $2.79 \pm 0.14$ & $3.17 \pm 0.16^{c}$ & $3.25 \pm 0.16^{\mathrm{B}}$ \\
\hline$P_{i}(\mathrm{mmol} / \mathrm{l})$ & $2.35 \pm 0.04$ & $2.61 \pm 0.07$ & $2.61 \pm 0.15$ & $2.65 \pm 0.20$ \\
\hline$\gamma A T P / P_{i}$ & $1.21 \pm 0.07$ & $1.07 \pm 0.04$ & $1.26 \pm 0.10$ & $1.35 \pm 0.17$ \\
\hline
\end{tabular}

Data represent the mean \pm SEM. $n=12 .{ }^{A} P<0.01,{ }^{B} P<0.05$, and ${ }^{\mathrm{C}} P=0.066$, for -120 minutes compared with 240 minutes; ${ }^{D} P=0.085$, for the mean difference from -120 to 240 minutes between groups. 
A
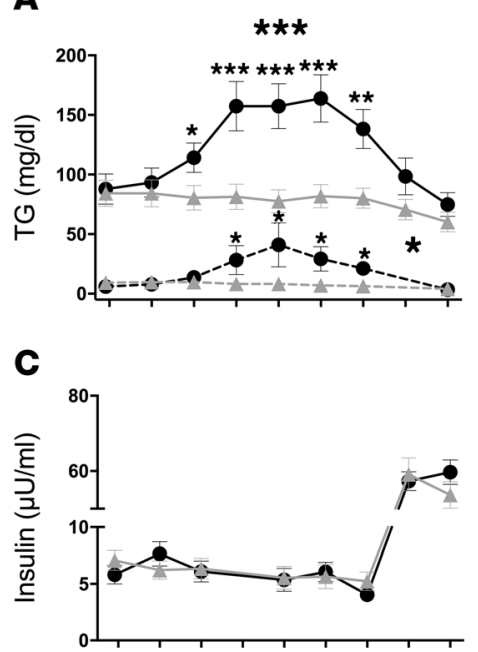

E

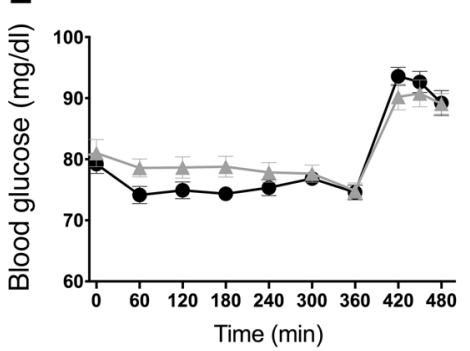

B

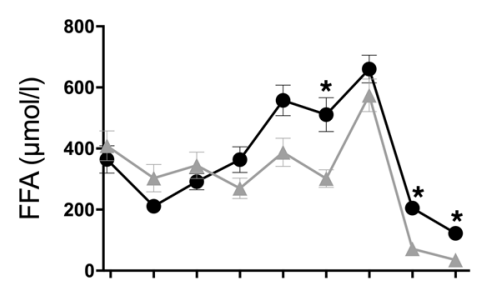

D

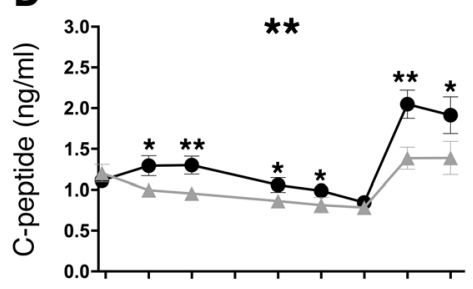

$\mathbf{F}$

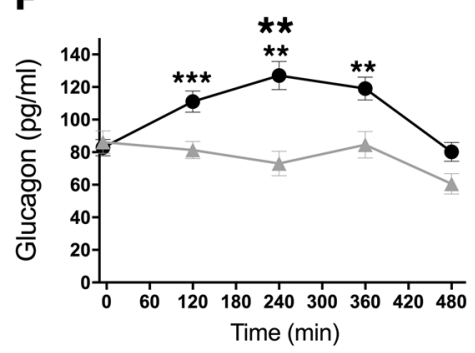

Figure 2. Time courses of circulating metabolites and hormones in humans. VCL (gray triangles) or PO (black circles) was administered at 0 minutes to lean, healthy men, and the hyperinsulinemic-euglycemic clamp was started at 360 minutes. TC circulating in plasma (solid line) and in chylomicrons (dashed line). The AUC was 59\% and $156 \%$ higher after PO ingestion, respectively (A). Circulating FFA (B). Time courses for insulin (C), C-peptide (AUC 28\% higher after PO) (D), blood glucose (E), and glucagon (AUC 41\% higher after PO) (F). Values represent the mean \pm SEM. $n=14$; chylomicron TC $n=6$. ${ }^{* *} P<0.001,{ }^{* *} P<0.005$, and ${ }^{*} P<0.05$, by paired, 2 -tailed $t$ test. Large asterisks refer to AUC differences; small asterisks refer to differences per time point.

Except for LPS, the analysis did not reveal whether the pathways and upstream regulators were activated or inhibited.

Under insulin- and noninsulin-stimulated conditions, PO resulted in upregulation of the following transcripts: miR1970, LPS-regulated genes (Ifit3, Clec4a3, Slc22a3, and C3ar1) (21-24), GOs2, and Ar16, while Tweak gene expression was downregulated. Opposing regulatory transcripts, i.e., those that were upregulated under insulin-stimulated conditions and downregulated under noninsulin-stimulated conditions, were also found and included, for example, transcripts involved in cell growth (Map3k13 and Slc3Oaf) $(25,26)$ and FFA metabolism and development of NAFLD (fatty acid-binding protein 5 or Fabp5) (27), as well as the predicted pseudogenes Gm3601 and H2-K2 (Figure 8E).

\section{Discussion}

This study demonstrates that a single oral dose of saturated fat increases hepatic TG accumulation, insulin resistance, GNG, and ATP concentrations in the human liver. Ingestion of saturated fat also induces peripheral insulin resistance in skeletal muscle and adipose tissue. In mice, a single saturated fat load preferentially induces hepatic insulin resistance and also affects hepatic gene expression and signaling, which could contribute to the promotion of NAFLD.

PO induced a marked increase in plasma FFA concentrations in both humans and mice, but no alterations in circulating inflammatory markers or adipokines, such as TNF- $\alpha$, IL- 6 , fetuin A, chemerin, and omentin. This finding indicates that the acute effects of $\mathrm{PO}$ are mediated by metabolic rather than endocrine changes and is partly in line with findings obtained after intravenous infusion of soybean oil, which showed no changes in circulating cytokines (19), or after ingestion of cream, which resulted in increased expression of TNF- $\alpha$ but not IL-6 (28). Interspecies differences could also be due to the administration of emulsified versus pure PO.

Notably, a single dose of PO markedly altered hepatic glucose fluxes and resulted in increased rates of GNG, reduced GLYnet, and a corresponding trend toward reduced GP flux. This finding extends our previous results on the effects of parenteral administration of polyunsaturated lipids (29). The present study also provides a comprehensive analysis of in vivo hepatic glucose and glycogen fluxes in humans that includes an assessment of glycogen cycling, which ensured correct estimation of GLY and GNG contributions to EGP (30). In chronic insulin-resistant states, such as occurs in T2DM or type 1 diabetes mellitus (T1DM), elevation of GNG and EGP coexists with enhanced glycogen cycling (30). Surprisingly, oral lipid loading stimulated GNG and hepatic insulin resistance without affecting glycogen cycling, which remained negligible, as was reported for healthy humans in the fasted state $(30,31)$. This indicates that healthy humans can rapidly downregulate GLY under conditions of elevated GNG to avoid futile cycling. The absence of any effect on glycogen cycling might be due to the prevailing euglycemia and basal peripheral insulinemia compared with the hyperglycemic hypoinsulinemic conditions in the aforementioned study. Consequently, the augmented glycogen cycling observed in insulin-resistant states reflects chronically abnormal hepatic energy metabolism rather than an immediate physiological response to changes in dietary lipid supply. Of note, these changes occur in the presence of higher glucagon concentrations, which are likely due to FFA-induced glucagon stimulation (32). Specifically, the increase in GNG and the decrease in GLYnet upon PO administration could, at least in part, result from the increase in circulating glucagon. Even small changes in plasma glucagon can modify GLYnet independently of insulin in healthy humans (33).

Interestingly, ingestion of PO increased hepatic $\gamma$ ATP and HCL concentrations, along with the induction of LOX and insulin resistance. Recently, we reported that a test involving ingestion of a mixed-meal with $35 \%$ fat content increases hepatic $\gamma$ ATP exclusively in insulin-resistant obese humans but not in lean, nondiabetic or T2DM individuals (34). This suggests that the stimulatory effect of saturated fat on hepatic energy metabolism is dose dependent and may be linked to the onset of insulin resistance, but not the insulin sensitivity state per se. In line with this, recent studies report the 
A

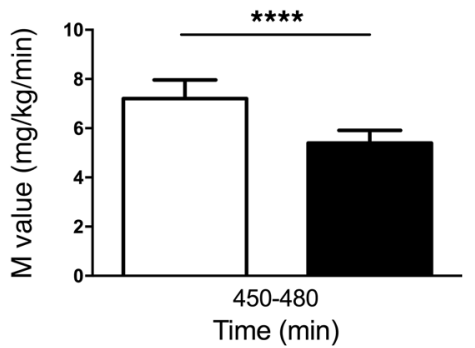

C
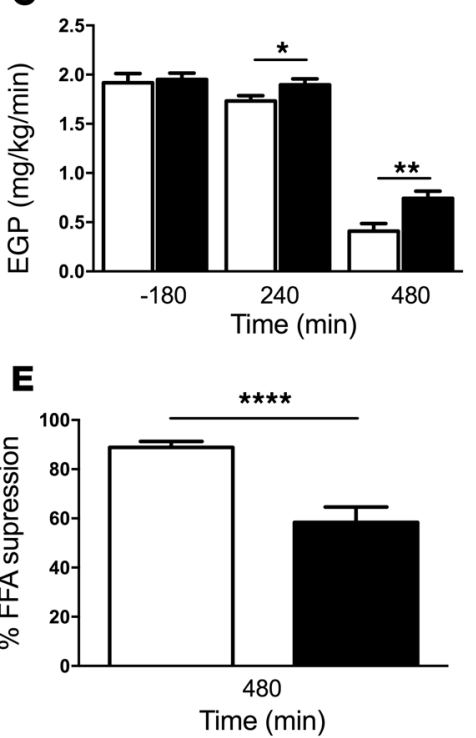

B

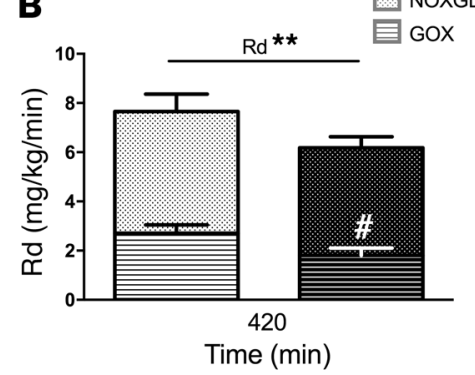

D

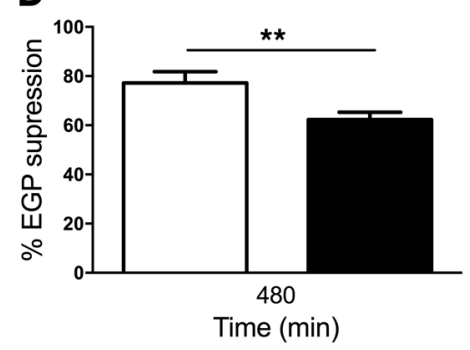

$\mathbf{F}$

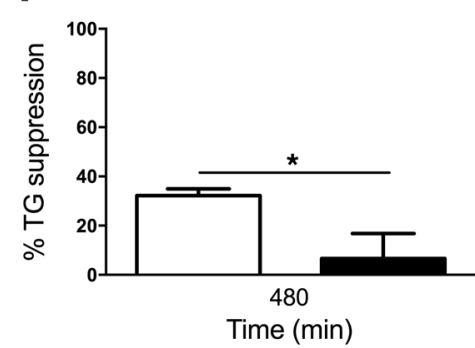

Figure 3. Parameters of insulin resistance in human volunteers after VCL or PO during clamp experiments. VCL, white bars; $\mathrm{PO}$, black bars. (A) WBIS, reflected by the M value. (B) Rd and its components GOX and nonoxidative glucose disposal (NOXCD). (C) EGP denoting hepatic insulin sensitivity at baseline (-180 min), after PO or VCL ingestion (240 $\mathrm{min}$ ), and under insulin-stimulated conditions during the clamp (480 $\mathrm{min}$ ). (D) Insulin-induced EGP suppression as an indicator of hepatic insulin sensitivity. (E) Insulin-induced FFA suppression reflecting adipose tissue insulin sensitivity and (F) the percentage of insulin-induced TC suppression. Data shown represent the mean $\pm S E M$. $n=14$. ${ }^{* * * *} P=0.0005$, ${ }^{* *} P<0.01$, and ${ }^{*} P<0.05$; ${ }^{*} P<0.05$, for GOX PO versus VCL. $P$ values were determined by 2 -tailed $t$ test and ANOVA.

upregulation of hepatic mitochondrial capacity in obese, insulinresistant, but not nondiabetic, humans in the absence of progressive NAFLD (35) and that T2DM patients have decreased ATP turnover associated with increased HCL (36). Collectively, these data suggest that an acute rise in lipid availability and oxidation can upregulate $\gamma$ ATP production and HCL deposition in young, healthy individuals with conserved mitochondrial plasticity, even after the development of acute insulin resistance. The minor increase in HCL has to be considered in the context of lean, insulin-sensitive individuals. In this case, it is reasonable to assume that an increase in HCL after just 1 dose of $\mathrm{PO}$ probably contributes to altered hepatic metabolism. Furthermore, PO, like meal ingestion, probably induced individual time courses of increases, so that maximum $\gamma$ ATP increases may have been missed in the absence of continuous magnetic resonance spectroscopy (MRS) monitoring, which would have been impossible, given the current experimental design.

Upon PO ingestion, human volunteers developed generalized insulin resistance, while mice responded primarily with hepatic insulin resistance. This may have resulted from species differences in insulin-sensitive tissues and lipid flux-buffering capacities of adipose tissue. Under physiological conditions, adipose tissue can buffer fatty acid flux into the bloodstream, thereby avoiding excessive exposure to lipotoxic stimuli. This is accomplished by suppression of FFA and TG release as well as increased clearance of circulating TG (37). Under the present experimental conditions, the high-dose PO likely impaired all 3 mechanisms in humans and to some extent in mice. The higher plasma TG concentrations observed in our study could have been due to higher rates of intestinal lipid absorption, altered hepatic lipid handling, or lower insulin sensitivity of the adipose tissue, as reflected by reduced insulin-induced suppression of FFA. Humans and mice both featured impaired insulininduced TG suppression after PO ingestion. This is partly in line with findings in humans after a fat-free test meal, in which T2DM patients were also unable to suppress plasma TG in contrast to lean, healthy men (38). The inability of our cohort to suppress TG secretion may have therefore at least partly resulted from an altered apolipoprotein metabolism associated with insulinresistant states and with decreased insulin-mediated fatty acid trapping by adipose tissue (37). In order to maintain low levels of HCL, the liver can only make use of either LOX or of lipid export of apolipoproteins (39). Here, we show that a single dose of PO induces both mechanisms, but nonetheless leads to increased HCL content. Of note, these changes occurred in the face of increased C-peptide, but unchanged insulin concentrations. The mismatch between insulin and C-peptide concentrations is likely explained by increased insulin clearance and turnover. While lower concentrations of palmitate decrease receptor-mediated insulin degradation in rat hepatocytes, higher concentrations of palmitate concentrations enhance this degradation (40). This can also explain the lack of increased insulin concentrations in the face of increased GLP-1 and GIP. In humans, most studies on insulin extraction used infusions of mainly polyunsaturated lipids, which revealed either unchanged or lower splanchnic insulin extraction $(17,41)$. Likewise, oral intake of soybean oil containing $61 \%$ polyunsaturated fatty acids resulted in increased concentrations of both insulin and C-peptide (19).

The marked alterations in hepatic glucose fluxes in humans and the predominant hepatic insulin resistance in mice raise interest in the effects of saturated fatty acids on hepatic gene expression, which has been previously examined mostly upon exposure to polyunsaturated fatty acids (42). The present study showed that a single dose of PO differentially regulated the canonical pathways TWEAK and AHR. The AHR pathway promotes NAFLD via upregulation of fatty acid transport (42), which is in line with the observed upregulation of Fabp5 and increase in HCL content in humans. The TNF-related TWEAK is known to promote cell turnover homeostasis through the NF- $\mathrm{kB}$ and $\mathrm{p} 38$ MAPK pathways and could serve as a biomarker of obesity and T2DM (43). TWEAK was also found to be associated with reduced TG accumulation in pal- 


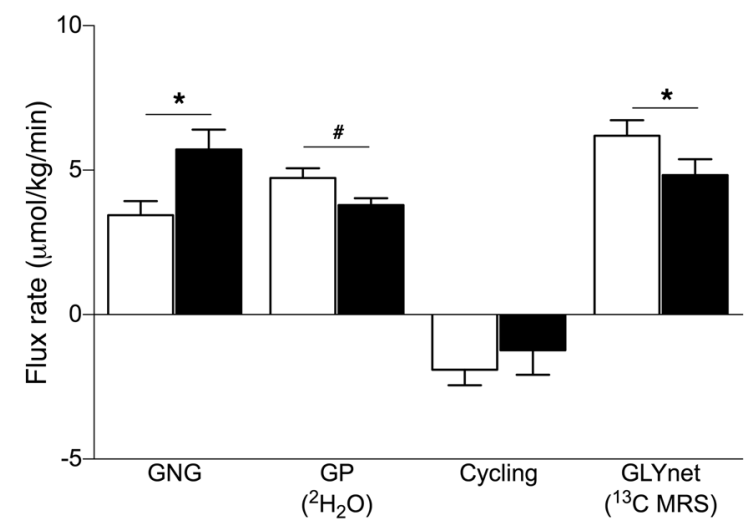

Figure 4. Hepatic glucose fluxes in humans. The rates of GNG, GP flux, glycogen cycling (cycling), and GLYnet were analyzed using in vivo ${ }^{13} \mathrm{C} /{ }^{31} \mathrm{P} /{ }^{1} \mathrm{H}$ and ex vivo ${ }^{2} \mathrm{H}$ MRS combined with ${ }^{2} \mathrm{H}_{2} \mathrm{O}$ ingestion, after either VCL (white bars) or PO (black bars) treatment, in lean, insulin-sensitive, male volunteers. Data represent the mean \pm SEM. $n=14$; GP and cycling $n=9$. ${ }^{*} P<0.05$ and ${ }^{\#} P=0.085$, by 2 -tailed $t$ test.

mitic acid-treated hepatocytes and may be involved in hepatic tissue repair (44). The present study cannot prove the activation status of TWEAK, thus making it difficult to interpret the observed downregulation of the Tweak gene and its receptor (Tnfrsf12a). However, recent findings of decreased circulating TWEAK concentrations correlating with obesity and concomitant NAFLD (45) could suggest that inhibition of the TWEAK pathway might increase susceptibility to hepatic injury.

The expression analyses further predicted PO-induced activation of LPS with or without insulin stimulation. This study also found upregulation of Ifit3, Clec4a3, Slc22a3, and C3ar1, genes regulated by LPS-stimulated macrophages. These data are in line with the reported increase in LPS concentrations upon high-fat ingestion $(28,46)$ resulting from lipid-induced disruption of the intestinal barrier (28). Interestingly, LPS is also known to decrease TWEAK signaling (47).

Downstream from LPS, NF- $\kappa$ B was found to be differentially regulated by $\mathrm{PO}$. Generally known for its proinflammatory properties, NF- $\kappa$ B is also an important antiapoptotic factor $(48,49)$. LPS activation and TNF collectively lead to increased TLR4 expression, proinflammatory cytokine production, and inflammation, on the one hand, and NF- $\mathrm{BB}$ activation and cytoprotection on the other (48). NF- $\kappa$ B activation leads to modest and short-lived JNK activation, in turn inducing antiapoptotic genes, such as c-FLIP (a caspase 8 inhibitor) and X-linked inhibitor of apoptosis (48). As a result, the active NF- $\mathrm{KB}$ pathway is critical for LPS-induced resistance to hepatotoxicity (50). Additionally, a high-fat diet and obesity are associated with prolonged JNK activation and TNFinduced cell death $(51,52)$. This alludes to an adaptation, which is lost upon repeated and/or sustained exposure to hepatotoxic stimuli leading to NAFLD and steatohepatitis. Furthermore, the constitutive activation of NF- $\kappa \mathrm{B}$ has been associated with severe hepatic and moderate peripheral insulin resistance (53). The present data suggest that a single PO challenge promotes pathways of LPSand TLR4-mediated inflammation and cytotoxicity, which are buffered by the activation of NF- $\kappa \mathrm{B}$, which in turn contributes to insulin resistance. This study also found altered regulation of other putative cytoprotective mechanisms including the phospholipase C4 pathway, which is important for hepatic regeneration (54), and PPAR $\alpha$, which serves as both a canonical pathway and an upstream regulator protecting against NAFLD progression (29).

Our analyses of the differential regulation of single genes by saturated fat revealed several genes of interest. The observed upregulation of GOs 2 may contribute to decreased TG clearance, thereby promoting NAFLD (55). The observed greater expression of Arl6 in the present study may also serve to protect against NAFLD,
$\mathbf{A}$

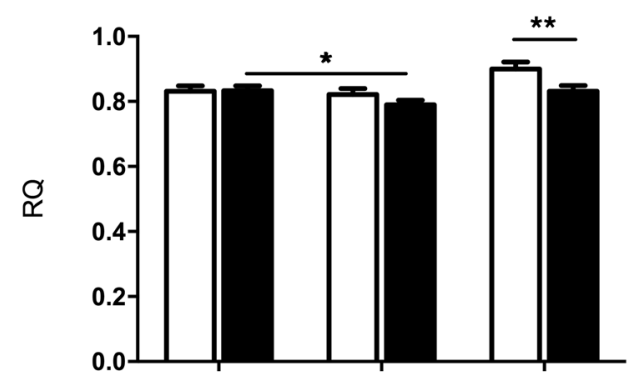

C

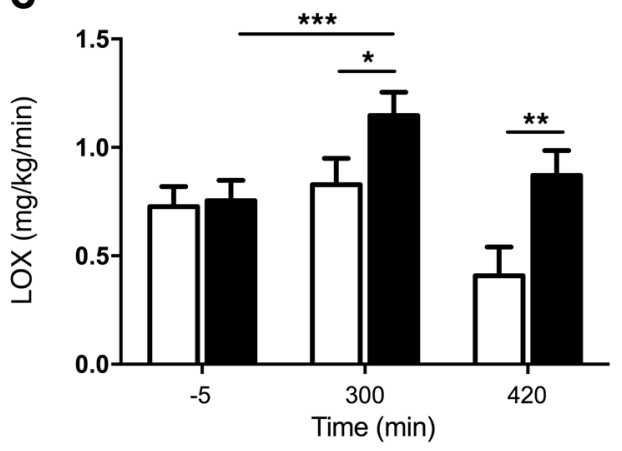

B

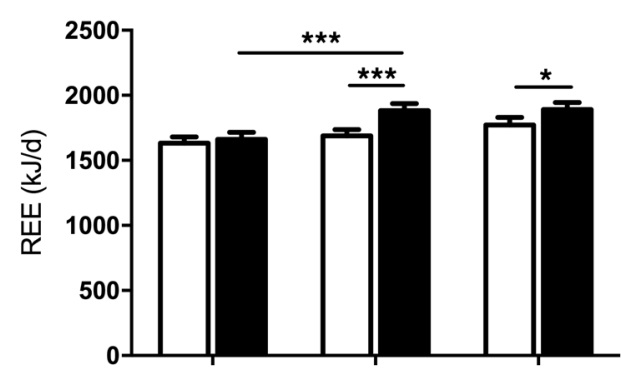

D

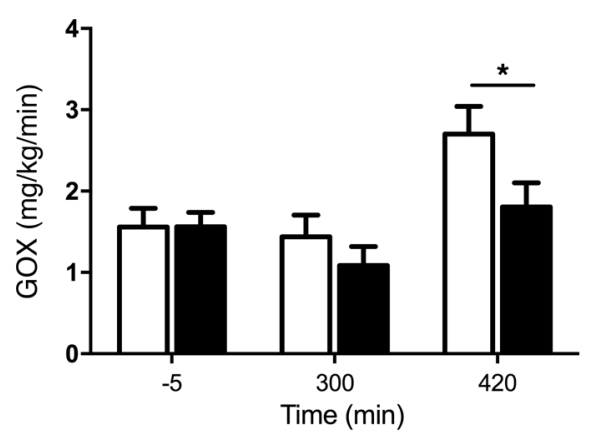

Figure 5. Time course of parameters of energy metabolism in lean, healthy volunteers after VCL and PO. VCL, white bars; PO, black bars. Parameters were obtained by performing an indirect calorimetry. The time points indicated are basal ( $-5 \mathrm{~min})$, 300 minutes after intervention, and 420 minutes under insulin-stimulated conditions. Effects on RQ (A), REE (B), LOX (C), and GOX (D). Data represent the mean \pm SEM. $n=14$. ${ }^{* * *} P<0.001$, ${ }^{*} P<0.005$, and ${ }^{*} P<0.05$, by ANOVA. 
A
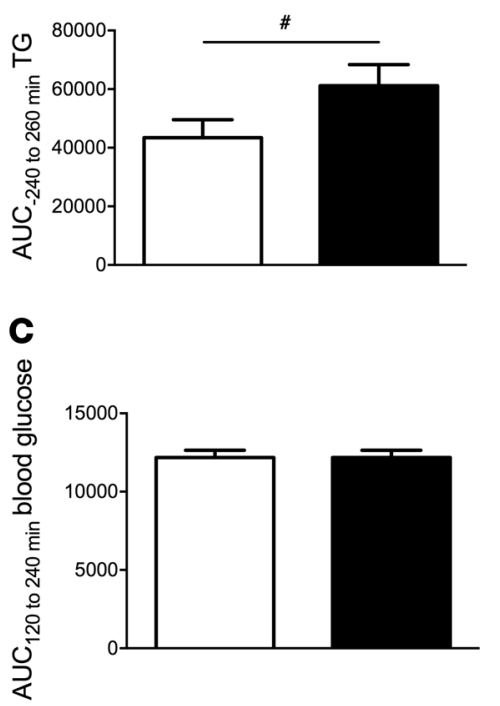

B

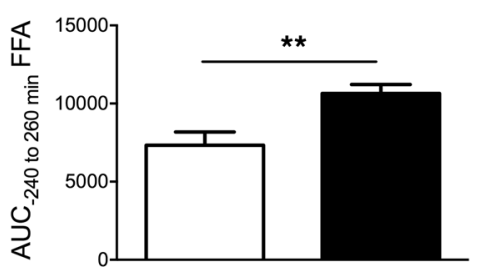

D

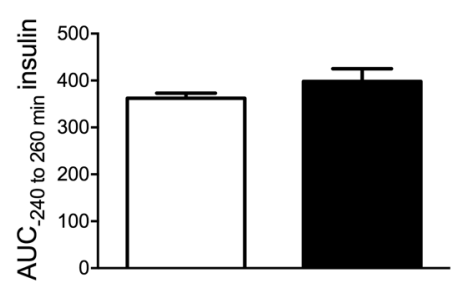

Figure 6. Circulating metabolites and hormones in mice. VCL (white bars) or PO (black bars) was administered via gavage to identical mouse cohorts at minute 0 , after a 6 -hour fast. Hyperinsulinemic-euglycemic clamp experiments were performed from 120 to 240 minutes. (A) The TC AUC tended to be higher after PO than after VCL. (B) The FFA AUC was increased after PO administration. Blood glucose (C) and insulin (D) levels were not different between groups. Data represent the mean \pm SEM. $n=6-10 .{ }^{* *} P<0.005$ and ${ }^{\#} P=0.08$, by 2 -tailed $t$ test. because loss of function of this gene has been implicated in obesity, NAFLD, and diabetes in Bardet-Biedl syndrome (56). Of note, the descriptive nature of the transcriptome analyses and expression profiling carried out in our study does not allow final conclusions to be drawn as to causality. That is to say, given the results of this study, it is impossible to single out 1 gene, transcription factor, or pathway that is activated by acute exposure to PO and that can be causally linked to either insulin resistance or steatosis.

This study offers the advantages of the translational approach in mice and humans, the use of near-physiologic administration of saturated fat, and the comprehensive phenotyping of in vivo hepatic glucose and energy metabolism. This study's limitations include the relatively small number of humans and mice, which might have prevented the detection of discrete effects of an acute lipid challenge. Nevertheless, the results demonstrate marked metabolic and transcriptional changes associated with PO treatment, even in these small groups. Another caveat of this study is that the hepatic expression data obtained in mice are not necessarily transferable to humans, as we could not obtain liver specimens from our human participants for ethical reasons. The lack of proteomic analysis of the targets identified by Ingenuity software constitutes another limitation of this study. Each pathway and regulator necessitates a thorough study of their role in the pathogenesis of insulin resistance and NAFLD, a task that must be tackled in subsequent studies.

The practical implication of this work is that the PO challenge used in this study most likely resembles the effects of ingestion of a meal rich in saturated fat, e.g., an 8-slice pepperoni pizza, containing approximately $16.72 \mathrm{~g}$ of saturated fat/110 $\mathrm{g}$ (57) or a meal consisting of a 110-g cheeseburger and a large portion of French fries, containing 18-25 g and 7-14 g per 1,000 kcal of saturated fat, respectively (58). One such meal would probably be sufficient to induce transient insulin resistance and impair hepatic metabolism, which necessitates the activation of hepatic defense mechanisms. Other simultaneously ingested biomacromolecules would exacerbate this metabolic challenge (59). The amount and types of fatty acids and carbohydrates in one such meal are in contrast to the diet recommendations of the American Diabetes Association (ADA). In this diet, daily intake of saturated fatty acids should not exceed $10 \%$ of total caloric intake. Furthermore, intake of monounsaturated fatty acids and carbohydrates from vegetables, fruits, whole grains, and legumes is recommended (60). We presume that lean, healthy individuals are able to compensate adequately for excessive intake of saturated fatty acids, however, sustained and repeated exposure to such nutrients will ultimately lead to chronic insulin resistance and NAFLD. Recent studies have shown hepatic energy metabolism alterations and induction of insulin resistance in obese and lean patients after ingestion of a simple hypercaloric mixed meal containing $24 \mathrm{~g}$ of fat or a drink containing $1 \mathrm{~g} / \mathrm{kg} \mathrm{BW}$ of a mixture of palmitate and soybean oil (34, 61,62 ). These results suggest that even lower doses of fatty acids are capable of inducing alterations similar to those observed with ingestion of pure $\mathrm{PO}$.

In conclusion, the initial effects of ingestion of saturated fat include (a) augmented hepatic energy metabolism and lipid storage; (b) impaired hepatic insulin sensitivity, along with increased GNG flux; and (c) altered hepatic expression of genes regulating inflammatory and protective pathways, which predispose to and protect against the development of NAFLD.

\section{Methods}

\section{Studies in humans}

Volunteers. Fourteen lean, young male volunteers were enrolled in this randomized, controlled crossover trial (Figure 1 and Figure 9). Participants were recruited from March 2012 through December 2013. The sample size calculation was based on a 2-sided, paired $t$ test, assuming a mean difference of EGP of 0.1 and a standard deviation of 0.11 , resulting in a sample size of 12 to reach a power of $80 \%$. The random allocation sequence was generated using SAS software (SAS Institute) by our in-house statistician. The possible order of treatments was randomly permuted in 2 blocks, with 1 extra block being generated to account for dropouts. Allocation was not concealed. Participants were enrolled and assigned to their treatment order by the study phy- 
A
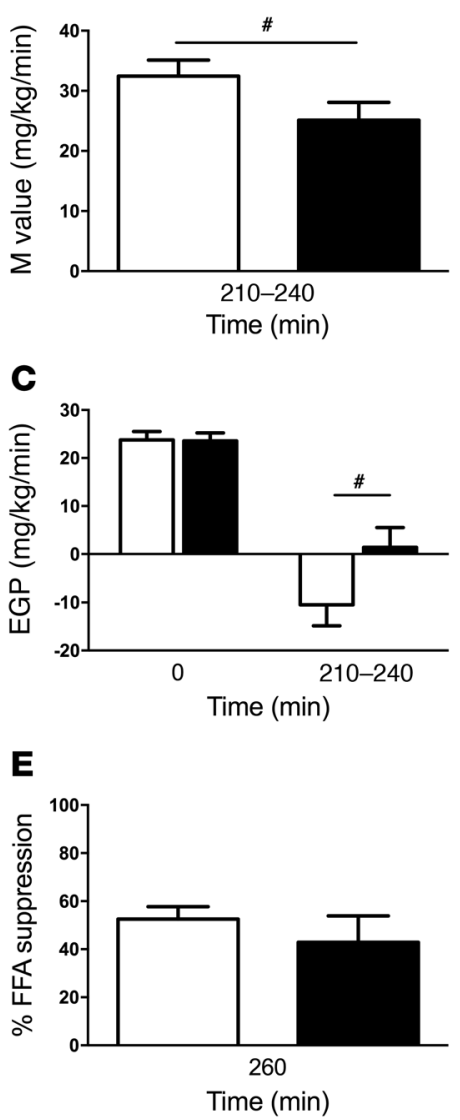

B
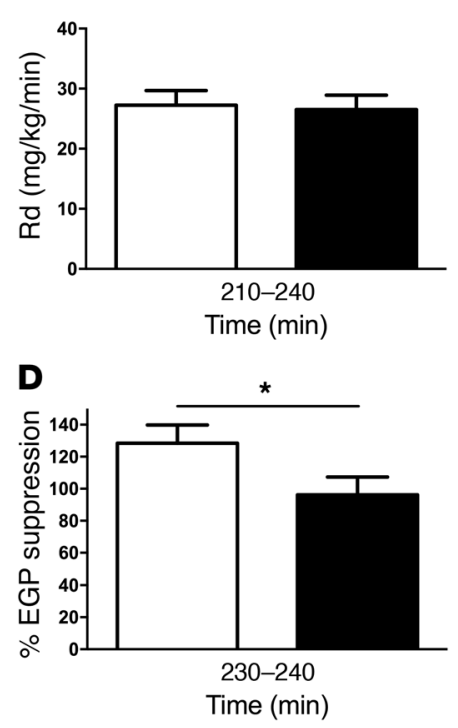

F

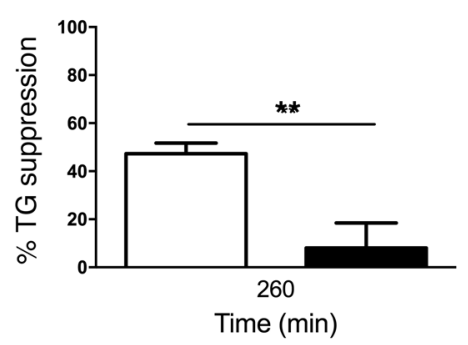

Figure 7. Parameters of insulin resistance in mice. VCL (white bars) or PO (black bars) was administered via gavage to identical mouse cohorts at minute 0 , after a 6-hour fast. Hyperinsulinemic-euglycemic clamp experiments were performed from 120 to 240 minutes. The $M$ value trended toward a reduction after $P O(A)$, while the Rd was unchanged (B). ECP at basal (0 min) and at clamp steady state (210-240 min) (C). EGP suppression was impaired after PO (D). Insulin-induced FFA suppression (E) and TC suppression $(\mathbf{F})$ are also shown. Data represent the mean \pm SEM. $n=9-10$. ${ }^{*} P<0.005$, ${ }^{*} P<0.05$, and ${ }^{\#} P=0.07$, by 2 -tailed $t$ test.

sician. Inclusion criteria were age between 20 and 40 years and a BMI between 20 and $25 \mathrm{~kg} / \mathrm{m}^{2}$. Exclusion criteria included dysglycemia, a family history of T2DM, acute, or chronic diseases, and the use of pharmacological agents known to affect insulin sensitivity, lipid metabolism, or immunological function. All participants underwent screening that included recording of clinical history, physical examination, bioimpedance assessment of lean body mass, routine laboratory tests, and a 75-g oral glucose tolerance test. Upon inclusion, participants were instructed to maintain their usual physical activity during the study period and to ingest a carbohydrate-rich diet for 3 days before each study day. They were then randomly assigned to 1 intervention and 8 weeks later to the other intervention. Basal hepatic ATP and HCL values from the study by Gancheva et al. were used as part of the control group data (63).

Experimental design. Participants arrived at the Clinical Research Center at $5 \mathrm{pm}$ and received a standardized dinner containing approximately $684 \mathrm{kcal}$ at 5:30 pm. At 8:00 pm, 10:00 pm, and 12:00 am, volunteers drank $1.6 \mathrm{~g} / \mathrm{kg}$ body water of ${ }^{2} \mathrm{H}_{2} \mathrm{O}$ (99.9\%; Sigma-Aldrich), up to a total dose of $5 \mathrm{~g} / \mathrm{kg}$ body water in order to assess GNG (19) (Figure 9). Body water was assumed to be $60 \%$ in all of our male study cohort

participants (30). Starting at 5:00 am (defined as time point -180 minutes) of the next day, participants drank $200 \mathrm{ml}$ of water containing $0.5 \%{ }^{2} \mathrm{H}_{2} \mathrm{O}$ every 60 minutes throughout the experiment to maintain an isotopic equilibrium of body water. At $-180,200$, and 400 minutes, participants received an oral dose of acetaminophen $(500 \mathrm{mg})$. For EGP calculation, participants received a 5 -minute priming bolus $(0.36 \mathrm{mg} / \mathrm{kg} \mathrm{BW} / \mathrm{min} \times$ fasting plasma glucose $[\mathrm{mg} / \mathrm{dl}])$ of D-[6,6- ${ }^{2} \mathrm{H}_{2}$ ]glucose (99\% enriched in ${ }^{2} \mathrm{H}$ glucose; Cambridge Isotope Laboratories) at -180 minutes, followed by a continuous infusion $(0.036 \mathrm{mg} / \mathrm{kg} \mathrm{BW} / \mathrm{min})$ (64). At zero time, participants drank either VCL or PO within 10 minutes. Patients with more than $70 \mathrm{~kg} \mathrm{BW}$ drank $92 \mathrm{~g}$, and those with less than $70 \mathrm{~kg}$ BW drank $80 \mathrm{~g}$ of PO $(\sim 1.18 \mathrm{~g} / \mathrm{kg}$ BW; Biopalm; Landkrone) (65). To facilitate ingestion, PO was heated to $60^{\circ} \mathrm{C}$, mixed with $1.84 \mathrm{~g}$ or $1.6 \mathrm{~g}$ emulsifier (Glice, Texturas; Albert y Ferran Adria), 9 or $8 \mathrm{~g}$ sugar-free vanilla syrup (Torani), and 81.2 or $70.4 \mathrm{ml}$ bottled still water, for a PO mix of $92 \mathrm{~g}$ and $80 \mathrm{~g}$, respectively. Oil test drinks were stirred constantly and served hot. For VCL administration, PO was substituted with $173.2 \mathrm{ml}$ or $150.4 \mathrm{ml}$ bottled still water, respectively. At 360 minutes, a hyperinsulinemiceuglycemic clamp began (10-min insulin bolus: $40 \mathrm{U} /$ hour; continuous insulin infusion: $40 \mathrm{mU} / \mathrm{m}^{2} / \mathrm{min}$; Insuman Rapid; Sanofi). Blood glucose concentration was adjusted to 90 $\mathrm{mg} / \mathrm{dl}$ by adapting the glucose infusion rate (GIR) using $20 \%$ glucose (B. Braun AG) enriched with $2 \% \mathrm{D}-\left[6,6-{ }^{2} \mathrm{H}_{2}\right]$ glucose, as described previously (19). Urine was sampled from -120 to 0 minutes, from 270 to 375 minutes, and from 390 to 510 minutes for the quantification of GNG and GLY. Blood was sampled at -60 and 360 minutes for assessment of GNG.

Indirect calorimetry. Indirect calorimetry (IC) was performed in the canopy mode using Vmax Encore 29n (CareFusion), as described previously (19), during baseline (at -170 min), intervention (at $200 \mathrm{~min}$ ), and steadystate clamp conditions (at $450 \mathrm{~min}$ ) for 20 minutes after a 10-minute adaptation period. RQ, REE, and substrate oxidation rates were calculated as reported previously (19). Nonoxidative glucose disposal was calculated from the difference between rates of glucose disappearance and carbohydrate oxidation.

Metabolites and hormones. Blood samples were immediately chilled, centrifuged, and the supernatants stored at either $-20^{\circ} \mathrm{C}$ or $-80^{\circ} \mathrm{C}$ until analysis. Venous blood glucose concentration was measured immediately using the glucose oxidase method (EKF Biosen C-Line glucose analyzer; EKF Diagnostics) (19). TG concentration was analyzed enzymatically on a Roche Cobas c 311 Analyzer (Roche Diagnostics). Serum chylomicron content was determined from the TG concentration in the first fraction of density-gradient ultracentrifugation (66). FFA were assayed enzymatically (Wako) using orlistat to prevent in vitro lipolysis (19). Serum C-peptide, insulin, and plasma glucagon levels were measured by radioimmunoassay (EMD Millipore). Cortisol levels in serum samples were measured by immunoassay using a Siemens Immulite 2000XPi Analyzer (67). GLP-1 and GIP were measured by ELISA (TECOmedical; EMD Millipore) (64). ELISA was used to measure plasma concentrations of IL-6, TNF- $\alpha$, fetuin-A (all using Quantikine HS ELISA kits from R\&D Systems), omentin, and chemerin (19) (both using ELISA kits from BioVendor). Intra- 
A
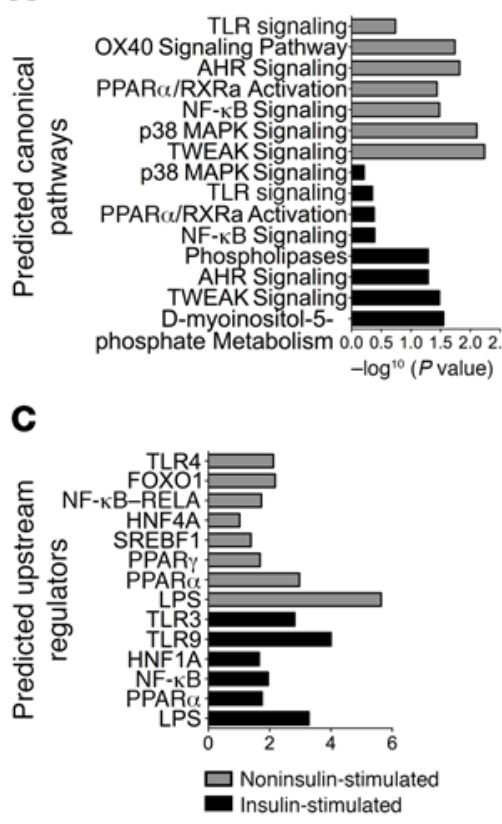

B
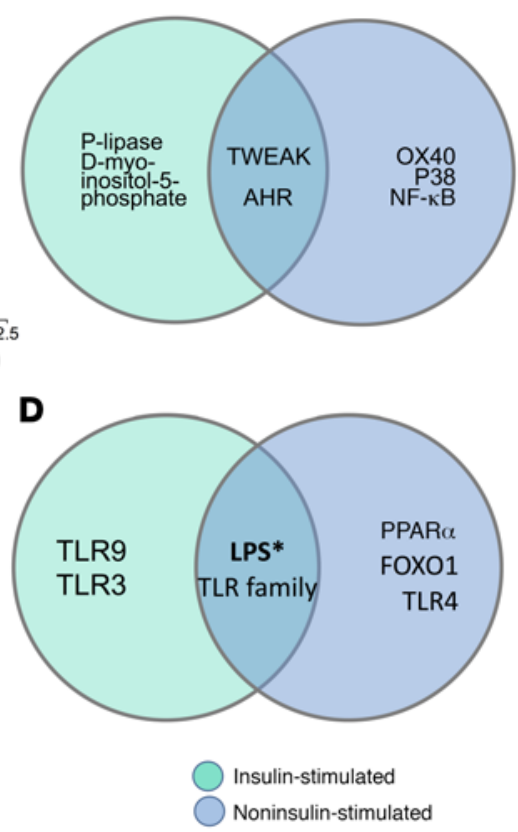

Figure 8. Transcriptome analysis after $\mathrm{PO}$ in murine hepatic tissue. Tissue was harvested from clamped and nonclamped murine cohorts. (A) Relevant canonical pathways predicted by Ingenuity software in hepatic samples from mice after PO under insulin- (black bars) and noninsulin-stimulated (gray bars) conditions. (B) Predicted canonical pathways with a $P$ value of less than 0.05 during insulin-stimulated (turquoise) and noninsulin-stimulated conditions (blue). (C) Noteworthy predicted upstream regulators and $(\mathbf{D})$ predicted upstream regulators with a $P$ value of less than 0.01 (asterisk denotes an activation $Z$ score above 1.9). (E) Genes that were upregulated in both cohorts (green), downregulated in both cohorts (yellow), and downregulated under insulin-stimulated, but upregu lated under noninsulin-stimulated, conditions (green and yellow, respectively) after PO treatment, with a $P$ value of less than 0.05 , a fold change greater than 1.3 , and an average expression in at least 1 group of greater than 4 ( $n=9-10,273$, and 327 probe sets, respectively).

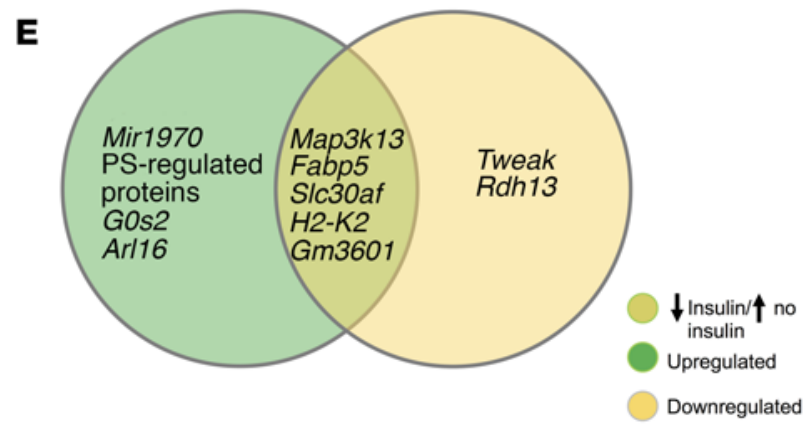

assay and interassay coefficients of variation (CV) for all cytokines were $2 \%$ to $7.2 \%$ and $4 \%$ to $14.4 \%$, respectively.

Glucose and glucuronide ${ }^{2} \mathrm{H}$ enrichment measurements by ex vivo ${ }^{2} \mathrm{H}$ MRS. The positional enrichment of urinary acetaminophen glucuronide and plasma glucose, resulting from ingestion of ${ }^{2} \mathrm{H}_{2} \mathrm{O}$ and acetaminophen at the level of glucose 6-phosphate (G6P), was assessed as previously described (30) to estimate the contributions of GNG and GLY to EGP. Plasma glucose was derivatized to monoacetone glucose (MAG), while urinary acetaminophen glucuronide was converted into 5-Oacetyl monoacetone glucuronic lactone (MAGLA) (68). When plasma glucose enrichment was inadequate, urinary glucuronide enrichment was analyzed instead, since both methods yield identical estimates of EGP contributions $(68,69)$. In total, 9 participants yielded sufficient data for NMR analysis, 5 of them for glucuronide. ${ }^{2} \mathrm{H}$ spectra were obtained with a Bruker Avance III HD 500 spectrometer equipped with a ${ }^{2} \mathrm{H}$-selective 5 -mm probe incorporating a ${ }^{19} \mathrm{~F}$ lock channel. For MAGLA samples, 5,000-10,000 free-induction decays (FIDs) were collected. For MAG samples, 20,000-40,000 FIDs were collected. Positional ${ }^{2} \mathrm{H}$ enrichments of MAG and MAGLA derived from plasma glucose and urinary anion gap (AG) were determined using the methyl signals as an intramolecular standard (30). All spectra were analyzed using the curve-fitting routine supplied with the NUTS PC-based NMR Spectral Analysis Program (Acorn NMR).
Gas chromatography-mass spectrometry. Determination of atom percentage enrichment (APE) of ${ }^{2} \mathrm{H}$ in blood glucose was done after deproteinization and derivatization to the aldonitrile-pentaacetate. The analyses were performed on a Hewlett-Packard 6890 gas chromatograph equipped with a $25 \mathrm{~m} / 0.25 \mathrm{~mm} / 0.12 \mu \mathrm{m}$ CPSil5CB capillary column (Chrompack; Varian) and interfaced with a Hewlett Packard 5975 mass selective detector. Selected ion monitoring was used to determine enrichments of fragments C3 to C6 (Supplemental Figure $1, \mathrm{~A}$ and B). Average mass units were 187 for endogenous glucose and 189 for D-[6,6- $\left.{ }^{2} \mathrm{H}_{2}\right]$ glucose as described previously (19).

MRS. All measurements were conducted with the volunteers lying in a supine position within a whole-body 3.0 T Achieva MRI machine (Philips Healthcare). Twelve volunteers were studied, including all participants for whom flux measurements were obtained. The effects of PO or VCL on HCL and hepatic ATP concentrations were assessed at baseline and 360 minutes after intervention. For hepatic ${ }^{1} \mathrm{H}$ MRS, a Q-body coil was used for shimming and HCL acquisition. Clinical T2-weighted turbo spin-echo (TSE) images were obtained in the transverse and coronal planes for localization and repositioning of the voxels used for HCL and ATP measurements. Respiratory-triggered ${ }^{1} \mathrm{H}$ spectra were acquired with a single-voxel $\left(30 \times 30 \times 20 \mathrm{~mm}^{3}\right)$ stimulated echo acquisition mode (STEAM) sequence. The variables were as follow: repetition time (TR) 3,000 ms, echo time $10 \mathrm{~ms}$, and signal averages 16 . To accu- 


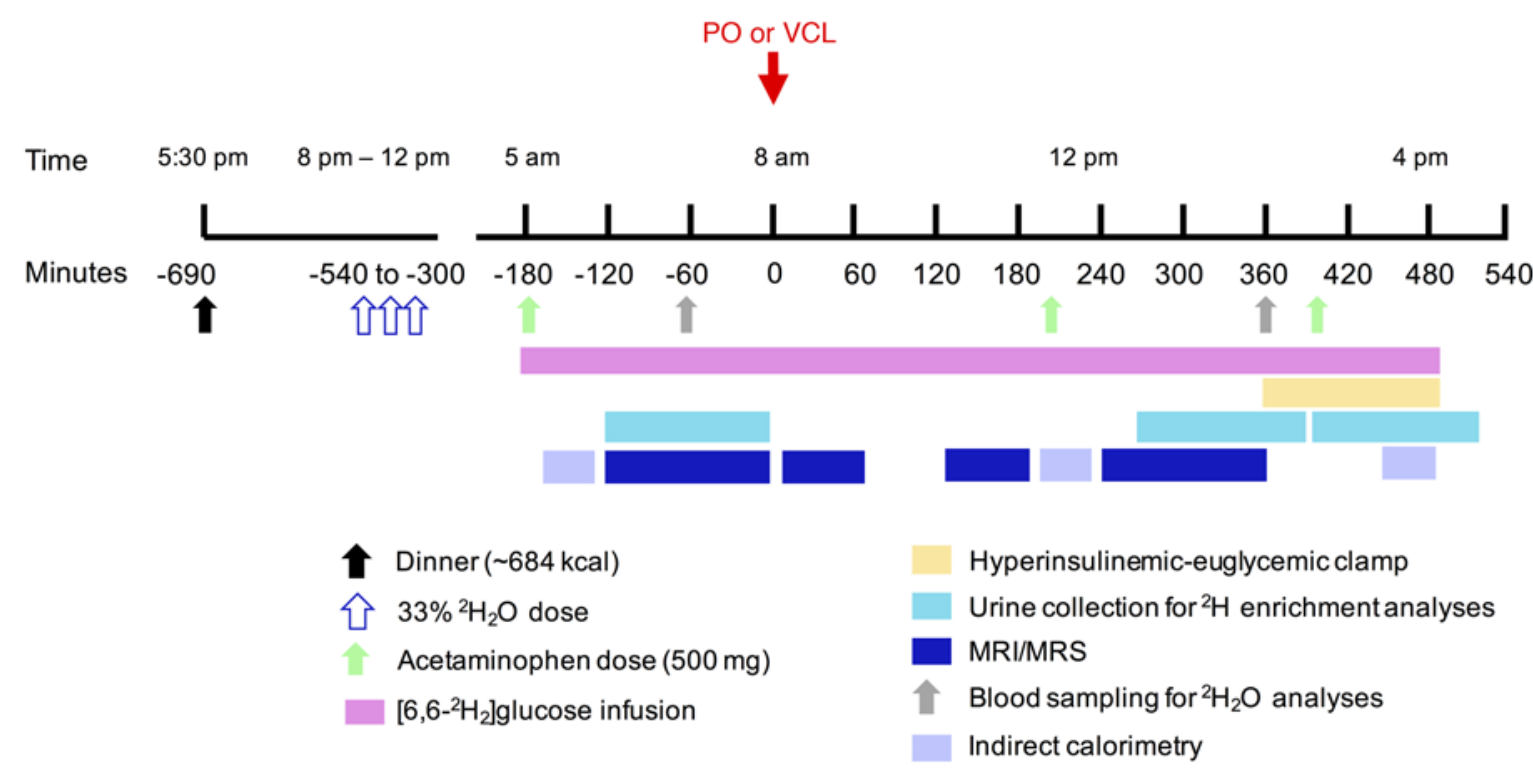

Figure 9. Human study design. Lean, healthy male adults randomly received either PO or VCL on 2 occasions. Hepatic metabolism was measured using in vivo ${ }^{13} \mathrm{C},{ }^{31} \mathrm{P},{ }^{1} \mathrm{H}$ and ex vivo ${ }^{2} \mathrm{H}$ MRS combined with ${ }^{2} \mathrm{H}_{2} \mathrm{O}$ and acetaminophen ingestion before and during hyperinsulinemic-euglycemic clamps with D- $\left[6,6-{ }^{2} \mathrm{H}_{2}\right]$ glucose-labeled $20 \%$ glucose infusion.

rately assess hepatic lipid volume, sets of non-water-suppressed and water-suppressed ${ }^{1} \mathrm{H}$ spectra were acquired, using a STEAM sequence (TR 3,000 ms, echo time $10 \mathrm{~ms}$, signal averages $16 \mathrm{~ms}$ ) and variable power and optimization relaxation (VAPOR) STEAM sequences (TR, echo time, and signal averages 3,000, 10, and $16 \mathrm{~ms}$, respectively). Water and lipid peaks were fitted and quantified using the NUTS software package (Acorn NMR), and lipid was expressed as the summation of the methyl and methylene fat peaks relative to water content using the equations described in ref. 70. For hepatic ${ }^{31} \mathrm{P}$ MRS, a $14-\mathrm{cm}$ ${ }^{31} \mathrm{P}$ circular surface coil (Philips Healthcare) was placed over the liver for the acquisition of hepatic ${ }^{31} \mathrm{P}$ spectra. Afterwards, ${ }^{31} \mathrm{P}-\mathrm{MRS}$ protondecoupled liver ATP measurements were conducted with a 3D imageselected in vivo spectroscopy (3D-ISIS) localized sequence (voxel: $60 \times 60 \times 60 \mathrm{~mm}^{3}$, TR: 6,000 ms, averages: 128, decoupling: WALTZ-4 [wideband alternating-phase low-power technique for zero-residual splitting -4], time: $13 \mathrm{~min})$. Absolute concentrations of $\gamma$ ATP (CV =9\%) and $\mathrm{Pi}(\mathrm{CV}=7 \%)$ were quantified using the jMRUI v4.0 software package (EC Human Capital and Mobility Networks, France), as described previously (19). Liver volume measurements were made from the coronal plane T2-weighted TSE images. For liver glycogen measurements, ${ }^{13} \mathrm{C}$ spectra were obtained with a 7-cm dual-tuned ${ }^{13} \mathrm{C} /{ }^{1} \mathrm{H}$ coil (PulseTeq Ltd.) (68), via a proton-decoupled pulse acquisition sequence (TR: 230 ms; bandwidth: $8 \mathrm{KHz}$, averages: $2 \times 4,000$; data points: 256; decoupling: continuous wave; time: $30 \mathrm{~min})$. Liver glycogen spectra were acquired with a block pulse ( $333 \mu \mathrm{s})$ that produced an Ernst angle at a distance of $35 \mathrm{~mm}$. Coil loading was corrected via integration of the right-most peak of a ${ }^{13} \mathrm{C}$-enriched sample of formic acid placed within the coil housing. Glycogen concentrations were determined from the integration of the C1-glycogen resonance (zero filling: 4k, effective line broadening: $20 \mathrm{~Hz}$ ) after the addition of 2 scans and baseline correction within NUTS software (Acorn NMR Inc.). The glycogen signal was corrected for distance and quantified via aqueous glycogen phantom measurements of 70 and $140 \mathrm{mmol} / \mathrm{l}$ measured at a distances of 15 to $37 \mathrm{~mm}$. The CV from repeated hepatic glycogen measurements was 6\%.
Calculations. Total AUC were calculated using the trapezoidal method. FFA and TG suppression was calculated from 100 - (clamp FFA or TG concentrations $\times 100$ )/basal FFA or TG concentrations, respectively. Glycogen cycling, i.e., simultaneous fluxes through glycogen synthase (GS) and GP, was assessed. In order to measure glycogen cycling, isotopic tracer measurements of EGP, GNG, and GP fluxes must be supplemented by a measurement of GLYnet (in this case, ${ }^{13} \mathrm{C}$ MRS) (71). GLYnet was calculated from the linear regression of hepatic glycogen content at -2, 0, 2, and 4 hours of PO or VCL ingestion using the least mean squares method. Rates of GLYnet were normalized to liver volume and BW and are expressed in $\mu \mathrm{mol} / \mathrm{kg} / \mathrm{min}$ (61).

Whole-body glucose disposal (M value) was calculated from glucose infusion rates during the clamp steady state. The rates of EGP (in $\mu \mathrm{mol} / \mathrm{kg} / \mathrm{min}$ ) were calculated by dividing the tracer infusion rate of $\mathrm{D}-\left[6,6-{ }^{2} \mathrm{H}_{2}\right]$ glucose times its enrichment to the hydrogens bound to carbon 6 , by the mean percentage of enrichment of plasma D- $\left[6,6-{ }^{2} \mathrm{H}_{2}\right]$ glucose and then subtracting the tracer infusion rate (19). To account for the incorporation of ${ }^{2} \mathrm{H}$ from ${ }^{2} \mathrm{H}_{2} \mathrm{O}$ by GNG during the overnight fast, background $\mathrm{D}-\left[6,6-{ }^{2} \mathrm{H}_{2}\right]$ glucose was determined before administration of ${ }^{2} \mathrm{H}_{2} \mathrm{O}$ as well as at -185 minutes on the day of the study. Consequently, for determination of basal EGP and EGP at the end of the intervention, the background $\mathrm{D}-\left[6,6-{ }^{2} \mathrm{H}_{2}\right]$ glucose enrichment from the -185-minute time point was used for calculations, whereas, during clamp conditions, with GNG being close to zero, the D-[6,6- $\left.{ }^{2} \mathrm{H}_{2}\right]$ value before administration of ${ }^{2} \mathrm{H}_{2} \mathrm{O}$ was used. GNG (in $\mu \mathrm{mol} / \mathrm{kg} / \mathrm{min}$ ) was calculated from the difference between EGP and GLYnet. The fractional GP flux contribution to EGP, i.e., the fraction of EGP originating from GLY, was calculated as 1 - (H5/ $\mathrm{H} 2$ ); where $\mathrm{H} 5 / \mathrm{H} 2$ is the ratio of glucuronide position 5 to position 2 enrichment from ${ }^{2} \mathrm{H}_{2} \mathrm{O}$, keeping in mind that glucose derived from GLY is enriched with ${ }^{2} \mathrm{H}$ in position 2, while glucose derived from GNG is enriched in positions 5 and 2. Absolute GP flux (in $\mu \mathrm{mol} / \mathrm{kg}$ / $\mathrm{min})$ was calculated from the equation: GP $=\mathrm{EGP} \times(1-\mathrm{H} 5 / \mathrm{H} 2)$. Glycogen cycling was then calculated as: GP - GLYnet. 


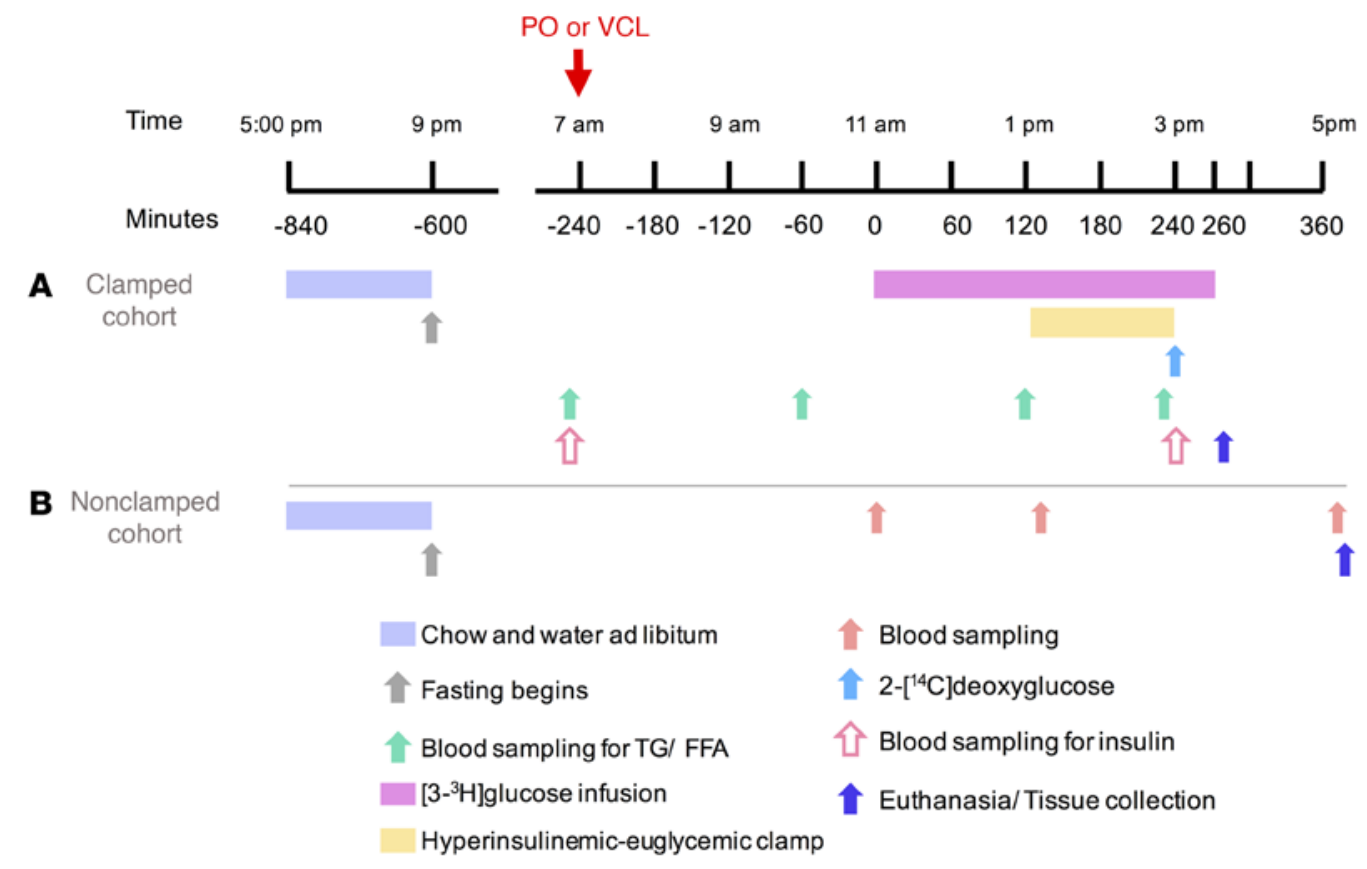

Figure 10. Mouse study design. Lean, adult male C57BL/6NTac mice were matched for BM and littermates and then divided into 2 cohorts. One cohort underwent hyperinsulinemic-euglycemic clamps after receiving either PO or vehicle via gavage (A), whereas another identical cohort underwent analysis of tissue and blood samples (B).

\section{Studies in mice}

Animals. Studies were conducted in lean, male, 14 week-old C57BL/6NTac mice (Taconic). Animals had ad libitum access to water and a standard chow diet. Mice were kept on a low-fat (LF) diet (13\% of calories derived from fat, $17 \mathrm{~kJ} / \mathrm{g}$, Standard Diet 1310; Altromin), and were matched for body mass (BM) and littermates. BM and composition (MiniSpec LF50; Bruker Optics) were measured 1 day prior to the start of the experiment. Animals were bred and housed in a temperature- and humidity-controlled environment including a 12-hour light/12-hour dark cycle, in compliance with the Federation of European Laboratory Animal Science Associations protocols.

Experiments under insulin-stimulated conditions. A permanent jugular vein catheter was placed under ketamine/xylazine anesthesia into a cohort of 19 mice with the aforementioned characteristics. Six to seven days later, the mice were fasted for 10 hours and then received $2 \mathrm{~g} / \mathrm{kg}$ BM PO or VCL via gavage. Six hours later, unrestrained, conscious mice underwent hyperinsulinemic-euglycemic clamps.

After 110 minutes of primed-continuous $\left[3-{ }^{3} \mathrm{H}\right]$ glucose infusion $(1.85 \mathrm{kBq} / \mathrm{min})$, a blood sample was collected to determine plasma insulin, glucose, and $\left[3-{ }^{3} \mathrm{H}\right]$ glucose concentrations for the calculation of basal EGP. A $\left[3-{ }^{3} \mathrm{H}\right]$ glucose infusion $(3.7 \mathrm{kBq} / \mathrm{min})$ containing insulin (15 pmol/kg/min; HumulinR; Lilly) was started. Blood glucose concentrations were measured every 10 minutes and target glycemia established by adjusting the GIR. At minute 120, 2-[14 C]deoxyglucose $(370 \mathrm{kBq})$ was injected intravenously to assess tissue-specific $\mathrm{Rg}$ rates. At the end of the experimental procedure, mice were euthanized by means of an intravenous ketamine/xylazine injection. Livers were collected, immediately snap-frozen in liquid nitrogen, and stored at $-80^{\circ} \mathrm{C}$. Blood was collected at culling, and plasma ${ }^{3} \mathrm{H}$ and ${ }^{14} \mathrm{C}$ radioactivity was determined in deproteinized plasma before and after ${ }^{3} \mathrm{H}_{2} \mathrm{O}$ evaporation to estimate glycolysis rates. In hepatic lysates, $2-\left[{ }^{14} \mathrm{C}\right]$ deoxyglucose-6-phosphate was separated from 2- $\left[{ }^{[4} \mathrm{C}\right]$ deoxyglucose via ion-exchange columns (Poly-Prep AG1-X8; Bio-Rad) as previously described (72). Glucose uptake was calculated by multiplying the mean plasma glucose levels between 120 and 140 minutes of the clamp $(\mathrm{mmol} / \mathrm{ml})$ by $2-\left[{ }^{14} \mathrm{C}\right]$ deoxyglucose tissue content $(\mathrm{dpm} / 100 \mathrm{~g}$ tissue), divided by the $2-\left[{ }^{14} \mathrm{C}\right]$ deoxyglucose plasma AUC in the same time frame. Radioisotopes were purchased from PerkinElmer and samples measured in an Ultima-Gold Scintillation Cocktail (TriCarb2910TR; PerkinElmer) (Figure 10). Whole-body glucose disposal ( $M$ value) was calculated from the tracer infusion rate, the specific activity of $\left[3-{ }^{3} \mathrm{H}\right]$ glucose, and BW.

Biochemical analyses. Blood glucose concentrations were assessed using a Contour hand-held glucometer (Bayer Vital). Plasma TG levels were determined by a colorimetric assay (Cayman Chemical), and plasma FFA levels were assessed with the FFA-HR(2)-Test (Wako). Hepatic TG levels were measured in whole-liver homogenates biochemically with the BioVision Assay.

Experiments under noninsulin-stimulated conditions. C57BL/6NTac mice $(n=20)$ with characteristics identical to those described above were fasted for 10 hours and given $2 \mathrm{~g} / \mathrm{kg}$ BM PO (Landkrone) or water (VCL) per gavage. Lateral tail vein blood samples were obtained prior to treatment and 2 hours afterward. Six hours after treatment, mice were euthanized with isoflurane, and a vena cava blood sample was collected and centrifuged at $4^{\circ} \mathrm{C}$, and plasma aliquots were immediately frozen in liquid nitrogen. Liver was dissected and immediately snap-frozen in liquid nitrogen (Figure 10).

\section{RNA isolation}

Snap-frozen liver samples from both cohorts were processed after administration of PO. Total RNA was isolated using the mRNeasy Mini Kit (QIAGEN). The Agilent 2100 Bioanalyzer was used to assess 
RNA quality, and only high-quality RNA (RNA integrity number [RIN] $>7$ ) was used for microarray analysis.

\section{Expression profiling}

Total RNA ( 30 ng) was amplified using the Ovation PicoSL WTA System V2 in combination with the Encore Biotin Module (both from NuGEN). Amplified cDNA was hybridized on Affymetrix Mouse Gene $2.1 \mathrm{ST}$ array plates containing approximately 35,000 probe sets. Staining and scanning (GeneChip Scanner 3000 7G; Affymetrix) were performed according to the Affymetrix Gene Titan expression protocol and modified according to NuGEN's Encore Biotin protocol.

\section{Statistical transcriptome analysis}

Expression Console software (version 1.3.0.187; Affymetrix) was used for quality control and to obtain annotated normalized robust multiarray average (RMA) gene-level data (standard settings included median polish and sketch-quantile normalization). Statistical analyses were performed using the statistical programming environment $\mathrm{R}$ implemented in CARMAweb (https://carmaweb.genome.tugraz.at/carma/). Genewise testing for differential expression was done using the limma $t$ test, and a $P$ value of less than 0.05 was set as the threshold to define sets of regulated genes. Filters for a fold change greater than 1.3 times and a linear average expression greater than 4 were applied. Pathway analyses were generated using the Ingenuity Pathway Analysis (QIAGEN; www.qiagen.com/ingenuity), where the overlapping $P$ value identifies transcriptional regulators that can explain observed gene expression changes. The activation $Z$ score helps infer activation states of predicted transcriptional regulators, with values of 2 or more indicating activation and values of -2 or less indicating inhibition.

\section{General statistical analyses}

Results are presented as the mean \pm SEM and were compared using a 2-tailed Student $t$ test or ANOVA adjusted for repeated measures, with Bonferroni's testing as appropriate. Calculations were performed using GraphPad Prism, version 6.02 (GraphPad Software). A P value of less than 0.05 was considered statistically significant, unless otherwise indicated.

\section{Study approval}

All participants provided written informed consent before inclusion in the study, which was performed according to the Declaration of Helsinki of 2013 and approved by the ethics board of the Heinrich Heine University Düsseldorf. All animal experiments were approved by the Upper Bavarian district government (AZ 55.2.1.54-2532-4-11).

\section{Author contributions}

MR initiated the investigation, led the clinical experiments and wrote, reviewed, and edited the manuscript. EÁH obtained and analyzed the data and wrote, edited, and reviewed the manuscript. SK obtained and analyzed the data, aided in designing the clinical study, and edited and reviewed the manuscript. AS obtained data and edited and reviewed the manuscript. PB designed the MRS study, obtained MRS data, and reviewed and edited the manuscript. EÁH, SK, AS, and PB contributed equally to this project. YK interpreted MRS data. $\mathrm{BN}$ researched the clinical data and reviewed and edited the manuscript. $\mathrm{CB}$ and FC performed derivatization experiments and enrichment analysis for ${ }^{2} \mathrm{H}-\mathrm{MRS}$. JGJ performed the ${ }^{2} \mathrm{H}-\mathrm{MRS}$ analyses and reviewed and edited the manuscript. PN conducted analyses and reviewed and edited the manuscript. $\mathrm{CH}$ conducted laboratory analyses and reviewed and edited the manuscript. SN and JR supervised the mouse studies and edited and reviewed the manuscript. MI analyzed transcriptomics data and edited and reviewed the manuscript. JB and MHdA supervised transcriptome analyses and edited and reviewed the manuscript. All authors gave final approval of the version to be published.

\section{Acknowledgments}

We thank Ulrike Partke and Anika Morcinietz at the German Diabetes Center (Düsseldorf, Germany) as well as Anke Bettenbrock, Jürgen Schultheiß, Moya Wu, and Anne Junker at the Helmholtz Center (Munich, Germany) for their excellent technical support. We also thank Alessandra Bierwagen at the German Diabetes Center for her guidance with MRS data analysis. This study was supported in part by the Ministry of Innovation, Science, and Research North Rhine-Westphalia (MIWF NRW); the German Federal Ministry of Health (BMG); as well as by a grant from the Federal Ministry of Education and Research (BMBF) to the German Center for Diabetes Research (DZD e.V.; DZD grant 2012), and by grants from the Helmholtz portfolio theme: Metabolic Dysfunction and Common Diseases, and the Helmholtz Alliance to Universities: Imaging and Curing Environmental Metabolic Diseases (ICEMED); the German Research Foundation (DFG; SFB 1116); the German Diabetes Association (DDG); and the Schmutzler-Stiftung. Financial support was also provided by the Portuguese Foundation for Science and Technology (research grant EXCL/DTP/0069/2012). Structural funding for the Center for Neurosciences and the UC-NMR facility was provided in part by the FEDER - European Regional Development Fund through the COMPETE Program; by grants from the Portuguese Foundation for Science and Technology (PEst-C/SAU/LA0001/2011, REEQ/481/QUI/2006, RECI/QEQQFI/0168/2012, and CENTRO-07-CT62-FEDER-002012); and by the Rede Nacional de Ressonância Magnética Nuclear.

Address correspondence to: Michael Roden, Auf'm Hennekamp 65, 40225, Düsseldorf, Germany. Phone: 49.0.211.33.82.201; E-mail: michael.roden@ddz.uni-duesseldorf.de.

BN's present address is: Clinical Research Services $\mathrm{GmbH}$, Mönchengladbach, Germany.

SN'S present address is: Sanofi-Aventis Deutschland $\mathrm{GmbH}$, R\&D Diabetes Research \& Translational Medicine, Frankfurt am Main, Germany.

\footnotetext{
1. van Dam RM, Willett WC, Rimm EB, Stampfer MJ, Hu FB. Dietary fat and meat intake in relation to risk of type 2 diabetes in men. Diabetes Care. 2002;25(3):417-424.

2. Mancini A, et al. Biological and Nutritional Prop-
}

erties of Palm Oil and Palmitic Acid: Effects on Health. Molecules. 2015;20(9):17339-17361.

3. Szendroedi J, et al. Abnormal hepatic energy homeostasis in type 2 diabetes. Hepatology. 2009;50(4):1079-1086.
4. Koliaki C, Roden M. Hepatic energy metabolism in human diabetes mellitus, obesity and nonalcoholic fatty liver disease. Mol Cell Endocrinol. 2013;379(1-2):35-42.

5. Erion DM, Shulman GI. Diacylglycerol-mediated 
insulin resistance. Nat Med. 2010;16(4):400-402.

6. Magkos F, et al. Effects of Moderate and Subsequent Progressive Weight Loss on Metabolic Function and Adipose Tissue Biology in Humans with Obesity. Cell Metab. 2016;23(4):591-601.

7. Winzell MS, Ahrén B. The high-fat diet-fed mouse: a model for studying mechanisms and treatment of impaired glucose tolerance and type 2 diabetes. Diabetes. 2004;53 Suppl 3:S215-S219.

8. Gastaldelli A, et al. Influence of obesity and type 2 diabetes on gluconeogenesis and glucose output in humans: a quantitative study. Diabetes. 2000;49(8):1367-1373.

9. Groop LC, et al. Glucose and free fatty acid metabolism in non-insulin-dependent diabetes mellitus. Evidence for multiple sites of insulin resistance. J Clin Invest. 1989;84(1):205-213.

10. Lewis GF, Carpentier A, Vranic M, Giacca A. Resistance to insulin's acute direct hepatic effect in suppressing steady-state glucose production in individuals with type 2 diabetes. Diabetes. 1999;48(3):570-576.

11. Bachmann OP, et al. Effects of intravenous and dietary lipid challenge on intramyocellular lipid content and the relation with insulin sensitivity in humans. Diabetes. 2001;50(11):2579-2584.

12. Roden M, et al. Mechanism of free fatty acidinduced insulin resistance in humans. J Clin Invest. 1996;97(12):2859-2865.

13. Szendroedi J, et al. Lipid-induced insulin resistance is not mediated by impaired transcapillary transport of insulin and glucose in humans. Diabetes. 2012;61(12):3176-3180.

14. Itani SI, Ruderman NB, Schmieder F, Boden G. Lipid-induced insulin resistance in human muscle is associated with changes in diacylglycerol, protein kinase C, and IkappaB-alpha. Diabetes. 2002;51(7):2005-2011.

15. Kashyap SR, et al. Discordant effects of a chronic physiological increase in plasma FFA on insulin signaling in healthy subjects with or without a family history of type 2 diabetes. Am J Physiol Endocrinol Metab. 2004;287(3):E537-E546.

16. Boden $\mathrm{G}$, et al. Effects of fat on insulinstimulated carbohydrate metabolism in normal men. JClin Invest. 1991;88(3):960-966.

17. Shah P, et al. Effects of free fatty acids and glycerol on splanchnic glucose metabolism and insulin extraction in nondiabetic humans. Diabetes. 2002;51(2):301-310.

18. Clore JN, Stillman JS, Li J, O'Keefe SJ, Levy JR. Differential effect of saturated and polyunsaturated fatty acids on hepatic glucose metabolism in humans. Am J Physiol Endocrinol Metab. 2004;287(2):E358-E365.

19. Nowotny B, et al. Mechanisms underlying the onset of oral lipid-induced skeletal muscle insulin resistance in humans. Diabetes. 2013;62(7):2240-2248.

20. Xiao C, Giacca A, Carpentier A, Lewis GF. Differential effects of monounsaturated, polyunsaturated and saturated fat ingestion on glucose-stimulated insulin secretion, sensitivity and clearance in overweight and obese, non-diabetic humans. Diabetologia. 2006;49(6):1371-1379.

21. Lee CG, Demarquoy J, Jackson MJ, O'Brien WE. Molecular cloning and characterization of a murine LPS-inducible cDNA. J Immunol.
1994;152(12):5758-5767.

22. Ban JY, Kim BS, Kim SC, Kim DH, Chung JH. Microarray Analysis of Gene Expression Profiles in Response to Treatment with Melatonin in Lipopolysaccharide Activated RAW 264.7 Cells. Korean J Physiol Pharmacol. 2011;15(1):23-29.

23. Li L, et al. A solute carrier family 22 member 3 variant $\mathrm{rs} 3088442 \mathrm{G} \rightarrow \mathrm{A}$ associated with coronary heart disease inhibits lipopolysaccharide-induced inflammatory response. J Biol Chem. 2015;290(9):5328-5340.

24. Sivapalaratnam S, et al. Identification of candidate genes linking systemic inflammation to atherosclerosis; results of a human in vivo LPS infusion study. BMC Med Genomics. 2011;4:64.

25. Masaki M, Ikeda A, Shiraki E, Oka S, Kawasaki T. Mixed lineage kinase LZK and antioxidant protein-1 activate NF-kappaB synergistically. Eur J Biochem. 2003;270(1):76-83.

26. Noh H, Paik HY, Kim J, Chung J. The alteration of zinc transporter gene expression is associated with inflammatory markers in obese women. Biol Trace Elem Res. 2014;158(1):1-8.

27. Westerbacka J, et al. Genes involved in fatty acid partitioning and binding, lipolysis, monocyte/ macrophage recruitment, and inflammation are overexpressed in the human fatty liver of insulinresistant subjects. Diabetes. 2007;56(11):2759-2765

28. Deopurkar R, et al. Differential effects of cream, glucose, and orange juice on inflammation, endotoxin, and the expression of Toll-like receptor-4 and suppressor of cytokine signaling-3. Diabetes Care. 2010;33(5):991-997.

29. Roden M, et al. Effects of free fatty acid elevation on postabsorptive endogenous glucose produc tion and gluconeogenesis in humans. Diabetes. 2000;49(5):701-707.

30. Kacerovsky M, et al. Postprandial and fasting hepatic glucose fluxes in long-standing type 1 diabetes. Diabetes. 2011;60(6):1752-1758.

31. Hundal RS, et al. Mechanism by which metformin reduces glucose production in type 2 diabetes. Diabetes. 2000;49(12):2063-2069.

32. Wang $\mathrm{L}$, et al. Acute stimulation of glucagon secretion by linoleic acid results from GPR40 activation and $[\mathrm{Ca} 2+] \mathrm{i}$ increase in pancreatic islet \{alpha\}-cells. J Endocrinol. 2011;210(2):173-179.

33. Roden M, et al. The roles of insulin and glucagon in the regulation of hepatic glycogen synthesis and turnover in humans. JClin Invest. 1996;97(3):642-648.

34. Fritsch M, et al. Time course of postprandial hepatic phosphorus metabolites in lean, obese, and type 2 diabetes patients. Am J Clin Nutr. 2015;102(5):1051-1058.

35. Koliaki C, et al. Adaptation of hepatic mitochondrial function in humans with non-alcoholic fatty liver is lost in steatohepatitis. Cell Metab. 2015;21(5):739-746.

36. Schmid AI, Szendroedi J, Chmelik M, Krssák M, Moser E, Roden M. Liver ATP synthesis is lower and relates to insulin sensitivity in patients with type 2 diabetes. Diabetes Care. 2011;34(2):448-453.

37. Frayn KN. Adipose tissue as a buffer for daily lipid flux. Diabetologia. 2002;45(9):1201-1210.

38. Søndergaard E, Sørensen LP, Rahbek I, Gormsen LC, Christiansen JS, Nielsen S. Postprandial VLDL-triacylglycerol secretion is not suppressed in obese type 2 diabetic men. Diabetologia. 2012;55(10):2733-2740

39. Perry RJ, Samuel VT, Petersen KF, Shulman GI. The role of hepatic lipids in hepatic insulin resistance and type 2 diabetes. Nature. 2014;510(7503):84-91.

40. Hennes MM, Shrago E, Kissebah AH. Receptor and postreceptor effects of free fatty acids (FFA) on hepatocyte insulin dynamics. Int JObes. 1990;14(10):831-841.

41. Wiesenthal SR, et al. Free fatty acids impair hepatic insulin extraction in vivo. Diabetes. 1999;48(4):766-774.

42. Vallim T, Salter AM. Regulation of hepatic gene expression by saturated fatty acids. Prostaglandins Leukot Essent Fatty Acids. 2010;82(4-6):211-218.

43. Maecker $\mathrm{H}$, et al. TWEAK attenuates the transition from innate to adaptive immunity. Cell. 2005;123(5):931-944.

44. Burkly LC. TWEAK/Fn14 axis: the current paradigm of tissue injury-inducible function in the midst of complexities. Semin Immunol. 2014;26(3):229-236.

45. Lozano-Bartolomé J, et al. Reduced circulating levels of sTWEAK are associated with NAFLD and may affect hepatocyte triglyceride accumulation. Int JObes (Lond). 2016;40(9):1337-1345.

46. Cani PD, et al. Metabolic endotoxemia initiates obesity and insulin resistance. Diabetes. 2007;56(7):1761-1772.

47. Chicheportiche Y, Fossati-Jimack L, Moll S, Ibnou-Zekri N, Izui S. Down-regulated expression of TWEAK mRNA in acute and chronic inflammatory pathologies. Biochem Biophys Res Commun. 2000;279(1):162-165.

48. Wullaert A, van Loo G, Heyninck K, Beyaert R. Hepatic tumor necrosis factor signaling and nuclear factor-kappaB: effects on liver homeostasis and beyond. Endocr Rev. 2007;28(4):365-386.

49. Chang $G$, et al. Hepatic TLR4 signaling is activated by LPS from digestive tract during SARA, and epigenetic mechanisms contribute to enforced TLR4 expression. Oncotarget. 2015;6(36):38578-38590.

50. Tang F, Tang G, Xiang J, Dai Q, Rosner MR, Lin A. The absence of NF-kappaB-mediated inhibition of c-Jun $\mathrm{N}$-terminal kinase activation contributes to tumor necrosis factor alpha-induced apoptosis. Mol Cell Biol. 2002;22(24):8571-8579.

51. Cai D, et al. Local and systemic insulin resistance resulting from hepatic activation of IKK-beta and NF-kappaB. Nat Med. 2005;11(2):183-190.

52. Jelenik T, et al. Tissue-specific differences in the development of insulin resistance in a mouse model for type 1 diabetes. Diabetes. 2014;63(11):3856-3867.

53. Santos SH, et al. Oral Angiotensin-(1-7) prevented obesity and hepatic inflammation by inhibition of resistin/TLR4/MAPK/NF- $\mathrm{BB}$ in rats fed with high-fat diet. Peptides. 2013;46:47-52.

54. Marion V, et al. BBS-induced ciliary defect enhances adipogenesis, causing paradoxical higher-insulin sensitivity, glucose usage, and decreased inflammatory response. Cell Metab. 2012;16(3):363-377.

55. Zhang X, Xie X, Heckmann BL, Saarinen AM, Czyzyk TA, Liu J. Targeted disruption of GO/ G1 switch gene 2 enhances adipose lipolysis, 


\section{CLINICAL MEDICINE}

alters hepatic energy balance, and alleviates high-fat diet-induced liver steatosis. Diabetes. 2014;63(3):934-946.

56. Fan Y, et al. Mutations in a member of the Ras superfamily of small GTP-binding proteins causes Bardet-Biedl syndrome. Nat Genet. 2004;36(9):989-993.

57. Haytowitz DB, Pehrsson PR, Holden JM. The National Food and Nutrient Analysis Program: A decade of progress. J Food Compost Anal. 2008;21(S1):S94-S102.

58. Urban LE, Roberts SB, Fierstein JL, Gary CE, Lichtenstein AH. Sodium, saturated fat, and trans fat content per 1,000 kilocalories: temporal trends in fast-food restaurants, United States, 2000-2013. Prev Chronic Dis. 2014;11:E228.

59. Teff KL, et al. Endocrine and metabolic effects of consuming fructose- and glucose-sweetened beverages with meals in obese men and women: influence of insulin resistance on plasma triglyceride responses. JClin Endocrinol Metab. 2009;94(5):1562-1569.

60. American Diabetes Association. 3. Foundations of Care and Comprehensive Medical Evaluation. Diabetes Care. 2016;39 Suppl 1:S23-S35.
61. Krssak M, et al. Alterations in postprandial hepatic glycogen metabolism in type 2 diabetes. Diabetes. 2004;53(12):3048-3056.

62. Beaudoin MS, Robinson LE, Graham TE. An oral lipid challenge and acute intake of caffeinated coffee additively decrease glucose tolerance in healthy men. J Nutr. 2011;141(4):574-581.

63. Gancheva S, et al. Variants in Genes Controlling Oxidative Metabolism Contribute to Lower Hepatic ATP Independent of Liver Fat Content in Type 1 Diabetes. Diabetes. 2016;65(7):1849-1857.

64. Jones JG, et al. NMR derivatives for quantification of ${ }^{2} \mathrm{H}$ and ${ }^{13} \mathrm{C}$-enrichment of human glucuronide from metabolic tracers. JCarbohydr Chem. 2006;25(2-3):203-217.

65. Nowotny B, Nowotny PJ, Strassburger K, Roden M. Precision and accuracy of blood glucose measurements using three different instruments. Diabet Med. 2012;29(2):260-265.

66. Herder C, et al. Adiponectin may mediate the association between omentin, circulating lipids and insulin sensitivity: results from the KORA F4 study. Eur JEndocrinol. 2015;172(4):423-432.

67. Brehm A, Krssak M, Schmid AI, Nowotny P, Waldhäusl W, Roden M. Increased lipid
The Journal of Clinical Investigation

availability impairs insulin-stimulated ATP synthesis in human skeletal muscle. Diabetes. 2006;55(1):136-140.

68. Jones J, Kahl S, Carvalho F, Barosa C, Roden M. Simplified analysis of acetaminophen glucuronide for quantifying gluconeogenesis and glycogenolysis using deuterated water. Anal Biochem. 2015;479:37-39.

69. Barosa C, Jones JG, Rizza R, Basu A, Basu R. Acetaminophen glucuronide and plasma glucose report identical estimates of gluconeogenesis and glycogenolysis for healthy and prediabetic subjects using the deuterated water method. Magn Reson Med. 2013;70(2):315-319.

70. Bischof MG, et al. Hepatic glycogen metabolism in type 1 diabetes after long-term near normoglycemia. Diabetes. 2002;51(1):49-54.

71. Kraegen EW, James DE, Jenkins AB, Chisholm $D J$. Dose-response curves for in vivo insulin sensitivity in individual tissues in rats. Am J Physiol. 1985;248(3 Pt 1):E353-E362.

72. Neschen S, et al. Prevention of hepatic steatosis and hepatic insulin resistance in mitochondrial acyl-CoA:glycerol-sn-3-phosphate acyltransferase 1 knockout mice. Cell Metab. 2005;2(1):55-65. 